\title{
Reconstrucción alométrica de la capacidad de vuelo de Pelagornis chilensis Mayr \& Rubilar-Rogers, 2010 (Aves: Pelagornithidae)
}

\author{
Iván MEZA-VÉLEZ
}

Departamento de Paleontología de Vertebrados, Museo de Historia Natural, Universidad Nacional Mayor de San Marcos. Lima 11, Perú; e-mail: imv2999@gmail.com

Meza-Vélez, I. 2020. Reconstrucción alométrica de la capacidad de vuelo de Pelagornis chilensis Mayr \& Rubilar-Rogers, 2010 (Aves: Pelagornithidae). [Allometric reconstruction of the flight capacity of Pelagornis chilensis Mayr \& Rubilar-Rogers, 2010 (Aves: Pelagornithidae)]. Spanish Journal of Palaeontology, 35 (2), 229-250.

Manuscript received 4 September 2019

Manuscript accepted 27 July 2020 https://doi.org/10.7203/sjp.35.2.18485

(C) Sociedad Española de Paleontología ISSN 2255-0550

\section{RESUMEN}

Las aves fósiles de la familia Pelagornithidae fueron grandes aves marinas planeadoras que habitaron desde el Paleoceno tardío hasta el Plioceno. En este trabajo se estudia la capacidad de vuelo de Pelagornis chilensis, una especie de esa familia. Utilizando la fórmula de sustentación y fórmulas alométricas se demostró que diferentes tipos de velocidades del ave extinta son significativamente mayores a las de Thalassarche chrysostoma, el albatros de cabeza gris, el ave marina actual que más se asemeja morfológicamente al ave extinta. Además, en coherencia con los resultados anteriores, se determinó que la potencia parásita, la potencia inducida, la fuerza muscular y el número de Reynolds de $P$. chilensis son significativamente mayores que las de T. chrysostoma. El método fue utilizado en el albatros Diomedea exulans para comprobar la cercanía de los valores observados con los calculados y estimar un porcentaje de error. La diferencia obtenida entre estos resultados es de un 2,7\%, demostrando la efectividad del método. Finalmente, se estudian las condiciones y forma de vuelo de los grandes albatros y su posible aplicación al vuelo de los Pelagornítidos. Se hipotetiza que por el gran tamaño de las especies estudiadas y por tener una morfología semejante, dichos patrones de vuelo serían similares.

Palabras clave: Pelagornithidae, albatros, vuelo, velocidad, alometría.

\begin{abstract}
Extinct birds of the family Pelagornithidae were large gliding seabirds that inhabited the planet from the late Paleocene to the Pliocene. This investigation studies the flight capacity of a species of pelagornithids, Pelagornis chilensis. Using the sustenance formula and allometric formulas it was shown that different speeds of the fossil bird are significantly greater than Thalassarche chrysostoma, the gray-headed albatross, which is the more morphologically close extant bird to $P$. chilensis. In addition, consistent with the previous results, it was determined that the parasitic power, the induced power, the muscular strength and the Reynolds number of P. chilensis are significantly greater than the same variables of $T$. chrysostoma. The method was used in the albatross Diomedea exulans, to check the proximity of the observed values with the calculated ones and estimate the error, obtaining a difference between these results of $2.7 \%$. Finally, the conditions and form of flight and their possible application to the flight are studied; it is hypothesized that due to the large size of both birds and morphology, these flight patterns would have been similar.
\end{abstract}

Keywords: Pelagornithidae, albatross, flight, speed, allometry. 


\section{INTRODUCCIÓN}

La familia Pelagornithidae (Fürbringer, 1888) es un grupo de grandes aves marinas (Mayr et al., 2008) con una distribución global, desde el Paleoceno tardío al Plioceno, en Eurasia, África, las Américas, Antártida, Japón y Nueva Zelandia (e.g., McKee, 1985; Olson, 1985, 1999; Tonni \& Tambussi, 1985; Averianov et al., 1991; Matsuoka et al., 1998; Olson \& Rasmussen, 2001; Walsh \& Hume, 2001; González-Barba et al., 2002; Rincón \& Stucchi, 2003; Bourdon, 2005). En América del Sur, existen en tres localidades: en la Formación Bahía Inglesa (norte de Chile) (Chavez \& Stucchi, 2002), en el extremo norte de América del Sur en el Mioceno de Venezuela (Rincón \& Stucchi, 2005), y en el Perú los primeros hallazgos de la familia Pelagornithidae son mencionados por Muizon \& DeVries (1985) en base a restos procedentes del Mioceno de la Formación Pisco (centro sur del Perú) (Chávez et al., 2007).

Estas aves se caracterizan por tener extensiones óseas espinosas del pico y la mandíbula denominados pseudodientes (Mayr \& Rubilar-Rogers, 2010; Feduccia, 1999; Ksepka, 2014). Poseían huesos altamente neumatizados, lo que dificulta el hallazgo de restos completos y preservados (Chavez et al., 2007) y fueron probablemente pelágicas, con alas largas y estrechas altamente especializadas para el planeo (Bourdon, 2005). La envergadura de las alas era aproximadamente el doble que la del albatros real actual (Ksepka, 2014). Comían en la superficie del agua, y sus "dientes" podían agarrar y sostener grandes presas (Bourdon, 2005). Las características de los huesos, como los pseudodientes, una mandíbula articulada y huesos especializados de las alas, han planteado interrogantes sobre la paleoecología y las afinidades filogenéticas de Pelagornithidae (Ksepka, 2014). Una característica importante de estas aves es su locomoción, estudios sobre su capacidad de vuelo afirman que los pelagornitidos eran aves planeadoras altamente eficientes con un amplio rango ecológico debido al planeo (Ksepka, 2014). El modelado de los parámetros de vuelo sugieren que tuvieron una estrategia de vuelo marino de largo alcance similar a la de los albatros existentes (Ksepka, 2014). También se ha estudiado el problema del vuelo de un ave tan grande como los Pelagornithidae, cuyo tamaño les habría ocasionado un gran estrés óseo de aleteo. Sin embargo, las simulaciones indican que esa tensión ósea se supera a una presión atmosférica de 1,3 bar (presión atmosférica actual, 1 bar), lo que implicaría un clima diferente al presente, que es consistente con los datos sobre las temperaturas globales promedio para el Mioceno (Cannell, 2020).

En este artículo se analiza la capacidad de vuelo del ave fósil Pelagornis chilensis como un problema de investigación, que será abordado con un método teórico, prescindiendo de un software de modelamiento. El objetivo principal del presente trabajo es determinar la capacidad de vuelo de $P$. chilensis, en términos de velocidad y otros parámetros aerodinámicos, con una metodología que aplica fórmulas físicas y relaciones alométricas tomando como referencia y objeto de comparación al albatros Thalassarche chrysostoma, justificado por su morfología similar con el ave extinta (Fig. 1). Además, se analizarán las características del vuelo del albatros como posibles características del vuelo de $P$. chilensis. Para ello se calcula, para $P$. chilensis, la velocidad mínima de vuelo, la velocidad de mínima potencia, la velocidad con el máximo rango, la velocidad con la tasa máxima de planeo, la velocidad de forrajeo asociada a una tormenta, la relación potencia parásita e inducida de $P$. chilensis con T. chrysostoma, y por fórmula la razón potencia parásita de $P$. chilensis/T. chrysostoma. Así mismo, se calcula cuántas veces la fuerza muscular de $P$. chilensis es la fuerza muscular de T. chrysostoma, el número de Reynolds de ambas aves y se comparan los modelos de flujo. Por último, para estimar el margen de error del cálculo alométrico, se calcula parámetros de velocidad de Diomedea exulans y se comparan los resultados con los valores observados de dicha ave. La importancia del presente trabajo radica en la metodología antes no empleada y en los parámetros de vuelo de un pelagornitido considerados por primera vez en este estudio, obteniendo resultados consistentes con lo esperado.

Una investigación comparable sobre el vuelo de un pelagornítido es el de Ksepka (2014), quien describe el holotipo de Pelagornis sandersi y estudia la capacidad de vuelo de esta ave extinta. Tanto la metodología como los parámetros aerodinámicos estimados son diferentes en ambos trabajos. Ksepka (2014) hace un modelamiento utilizando el software de vuelo Flight 1.25 con el que halla parámetros aerodinámicos de $P$. sandersi en términos de la relación entre la sustentación y la resistencia (arrastre). No obstante, el objetivo de Ksepka (2014) no es estimar la velocidad. Otra diferencia son las dimensiones y algunas características de las especies estudiadas; mientras que $P$. sandersi es el pelagornis de mayor envergadura $(\sim 6.4 \mathrm{~m})$, P. chilensis posee $\sim 5.2 \mathrm{~m}$ de la misma medida. A nivel óseo las diferencias morfológicas más significativas están en el cráneo: el de $P$. sandersi presenta una esbelta porción caudal de la mandíbula, en cambio, la misma región es cuadrada y profunda en $P$. chilensis. Esta diferencia se refleja en un pico más alargado y con mayor número de pseudodientes mandibulares (31 vs 20) en $P$. sandersi que en $P$. chilensis (Ksepka, 2014). Con respecto al vuelo, estas diferencias craneanas no son significativas. En cuanto al estado del material, el holotipo de $P$. chilensis está más completo y conservado (ver Materiales) que el holotipo de $P$. sandersi, que presenta el cráneo y la rama mandibular derecha, parcialmente la fúrcula, la escápula derecha, el húmero derecho sin extremo distal, extremos distal y proximal del radio derecho, fragmentos 


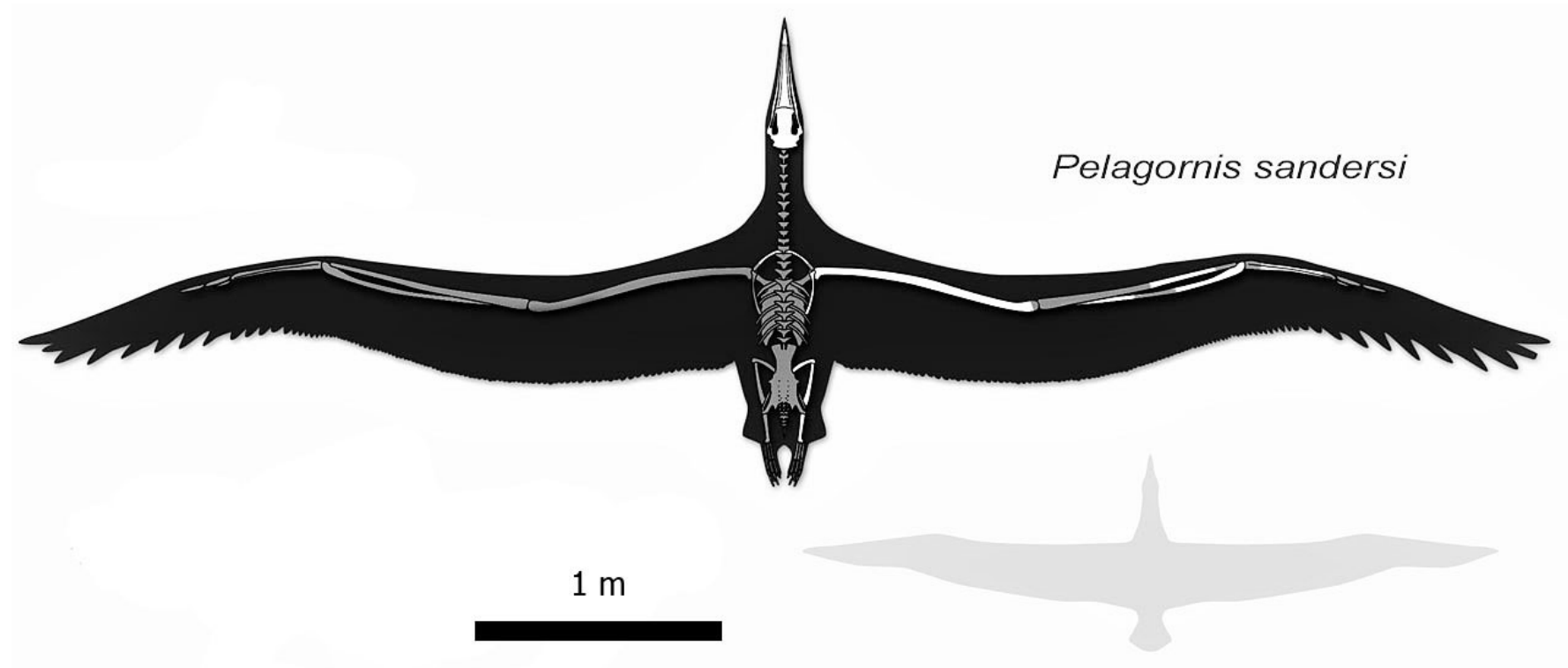

Royal Albatross

Figura 1. Pelagornis sandersi y el Albatros Royal. Imagen modificada de Liz Bradford.

de ulna, carpometacarpo, fémur derecho, tibiotarso, fíbula, tarsometatarso y una falange. Con el material descrito, en ambos trabajos se ha podido reconstruir la especie y estimar su envergadura. Además, teniendo pocos elementos, Ksepka (2014) ha obtenido resultados satisfactorios de la capacidad de vuelo de $P$. sandersi. En consecuencia, esta investigación también espera obtener valores estimados de parámetros aerodinámicos de $P$. chilensis cercanos a los reales.

Existen otros trabajos relevantes asociados al tema de esta investigación. Por ejemplo, Pennycuick (1975) desarrolla la mecánica de vuelo de las aves, que será la base teórica para el estudio alométrico del vuelo de albatros y petreles actuales en Pennycuick (2008) y para el estudio aerodinámico del vuelo de los Procellariiformes en Pennycuick (1987). Por último, Cannell (2020) utiliza el software Flight 1.25 para estudiar las limitaciones de vuelo en la atmósfera actual de las aves gigantes del Mioceno, Argentavis magnificens y los Pelagornithidae, tomando como base el trabajo de Pennycuick (1996), donde estudia el estrés y la tensión muscular en el vuelo de las aves, en particular en las aves gigantes. Para ello analiza el vuelo de Argentavis, que requeriría valores de tensión muy altos para un vuelo nivelado, a menos que la densidad del aire fuera más alta en el Mioceno que en la actualidad, o la gravedad menor. En una publicación anterior, este investigador ya había abordado la problemática del vuelo de un ave de gran tamaño como Argentavis y el vuelo planeado del albatros Diomedea exulans (Pennycuick, 1986).

El albatros de cabeza gris, Thalassarche chrysostoma, escogido para esta investigación es un ave marina de gran tamaño perteneciente a la familia Diomedeidae, orden Procellariiformes. Un carácter compartido con los petreles gigantes (Macronectes spp.) es que el húmero extendido puede ser "bloqueado" por un abanico de tendones que impide que el ala se eleve por encima de la horizontal (Brooke, 2002). Una vez que el húmero se retrae ligeramente de la posición totalmente hacia adelante, la cerradura ya no funciona y el ala se puede elevar, facilitando notablemente el planeo del albatros (Pennycuick, 1982). Los adultos pesan aproximadamente 3-4 kg, tienen una envergadura de poco más de $2 \mathrm{~m}$ (Pizzey \& Knight, 1999) y la longitud del cuerpo, desde la punta de la cola hasta la punta del pico, es aproximadamente de 70-85 cm (Schulenberg et al., 2010).

Los albatros vuelan largas distancias sobre el Océano Sur casi sin batir sus alas (Croxall et al., 2005; Safina, 2002, 2007). El vuelo sostenido durante tanto tiempo sin batir las alas ha intrigado a los observadores que especulan sobre cómo el albatros puede extraer energía del viento (Richardson, 2011). Dos teorías se han propuesto para el vuelo sin aleto de estas aves. La primera propone que el albatros usa cizalladura (o corte) del viento para ganar energía, es decir, el aumento de la velocidad del viento con la altura sobre la superficie del océano (ascenso vientocizalladura). La segunda teoría propone que un albatros utiliza corrientes ascendentes causadas por el viento que sopla sobre las olas para obtener energía (ascenso ola-pendiente). La primera teoría tiene más respaldo (Richardson, 2011). Richardson (2011) afirma que el viento que sopla sobre las olas tiene simultáneamente cizalladura del viento y movimientos verticales. Además, el viento que interactúa con las olas frecuentemente contiene ráfagas y 
remolinos, que tienen corrientes ascendentes y cizalladura del viento. A partir de las olas generadas por el viento, dado un tiempo suficiente, estas conviven con el viento en cizalla en mar abierto. El investigador sostiene que en el Océano Sur, donde los albatros se elevan más, hay fuertes vientos y grandes olas, incluyendo tanto las olas generadas por el viento localmente como olas de oleaje generadas en otra parte.

El estudio de aves extintas ha sido abordado utilizando otras herramientas metodológicas a las ya expuestas, es el caso de las aves del Cretácico. Serrano \& Chiappe (2017) usan una combinación de modelado computacional y análisis morfofuncional (análisis multivariante) para inferir las propiedades de vuelo de Sapeornis chaoyangensis, un ave del Cretácico temprano del tamaño de un cuervo. El análisis morfofuncional correlaciona la longitud de la cresta deltopectoral (DPC) del humero con la frecuencia del batir del ala. Esto implica que, para una determinada masa corporal (BM), un pájaro con un DPC más corto tiene ventajas mecánicas para batir sus alas a velocidades más altas y, por lo tanto, más frecuencia sobre un pájaro con un DPC más largo (Serrano \& Chiappe, 2017). Por lo tanto, la relación entre BM y la longitud del DPC se puede usar para inferir el patrón de vuelo básico, es decir, aleteo versus planeo. Serrano \& Chiappe (2017) también investigan la eficiencia energética de $S$. chaoyangensis bajo vuelo de aleteo sostenido a través de modelos computacionales. Una de las consecuencias de la investigación de Serrano \& Chiappe (2017), relevante para este trabajo, es que hace 125 millones de años algunas aves ya habían desarrollado los requerimientos morfológicos y aerodinámicos para un tipo de vuelo planeado ("soaring") utilizando las térmicas continentales.

Otra investigación destacable en vuelo de aves fósiles, tanto por su metodología como por sus conclusiones, es el ofrecido por Serrano et al. (2017), en el cual se infieren parámetros de vuelo de aves no Neornithes del Mesozoico aplicando análisis multivariante de elementos de extremidades anteriores de sus parientes actuales (Neornithes). Los parámetros aerodinámicos relevantes en este trabajo son la carga del ala $(\mathrm{WL}=\mathrm{m} / \mathrm{S}$; donde $\mathrm{m}$ es la masa y $\mathrm{S}$ es la superficie horizontal del ala) y la relación de aspecto $\left(\mathrm{AR}=\mathrm{B}^{2} / \mathrm{S}\right.$; donde $\mathrm{B}$ es la envergadura). Ambos parámetros se relacionan con los costos de energía de la locomoción aérea y la maniobrabilidad del vuelo, donde $\mathrm{B}$ y S fueron obtenidos con modelos de regresión. Los resultados indican que los modos de vuelo más modernos (aleteo continuo, aleteo y planeo, aleteo intermitente, planeo térmico: "soaring") fueron posibles para la amplia gama de taxones aviares no Neornithes. Un estudio con una metodología similar es el de Serrano et al. (2018), el cual reconstruye los modos de vuelo de dos pequeños Enantiornithes, Concornis lacustris y Eoalulavis hoyasi, del Cretácico Inferior de Las Hoyas (Cuenca, España).
El vuelo de los animales se puede clasificar en tres tipos principales: el vuelo en paracaídas, el planeo y el vuelo batido (aleteo). El vuelo de los pelagornítidos y de los albatros es principalmente el planeo. Durante el planeo, donde las alas están extendidas y estáticas, se consume muy poca energía. La mayoría de las aves planeadoras también pueden hacer el vuelo batido (Villar et al., 2013). Un ave voladora genera fuerzas de sustentación para contrarrestar la gravedad y fuerzas de empuje para vencer la resistencia. La magnitud de estas fuerzas se puede aproximar usando principios físicos elementales. El caso más simple es el vuelo estable o de crucero, es decir, en el aire en calma a una velocidad uniforme y a una altitud constante. Este caso requiere de fuerzas equilibradas, donde la elevación es igual al peso y el empuje es igual a la resistencia (Videler, 2005).

Las alas cumplen dos funciones: la sustentación, producida por el principio de Bernoulli, y la propulsión o empuje. En este caso, las alas se doblan hacia arriba y baten hacia abajo y hacia atrás (Villar et al., 2013). El empuje es la fuerza que genera el ave a velocidad uniforme para vencer la fuerza de resistencia del aire, que se encuentra con el ave y para que esta siga volando a la misma velocidad, debe generar una cantidad de empuje igual a la resistencia total sobre el cuerpo y las alas en la dirección horizontal. Para ello aletea o da golpes de ala acelerando el aire hacia atrás del ave (Videler, 2005). Además, el ángulo de ataque de las alas se ajusta a las necesidades del ave, según si quiere subir, bajar o volar horizontalmente (Villar et al., 2013). En el vuelo de un ave también intervienen los momentos (magnitud física relacionada a la rotación). Se asume que la suma de momentos de cabeceo hacia arriba y hacia abajo alrededor del centro de gravedad es cercana a cero y se puede despreciar (Videler, 2005).

Dos parámetros de vuelo importantes para la aerodinámica de un ave voladora son la carga alar y la relación de aspecto. Estos proporcionan una aproximación al modo en que un ave pueda volar con eficiencia (Serrano et al., 2017). La carga alar, que es el peso soportado por una unidad de superficie del ala, es directamente proporcional a la velocidad en las fases de no aleteo de vuelo e inversamente proporcional a la maniobrabilidad (Norberg, 2002; Longrich, 2006; Pennycuick, 2008; Tennekes, 1996). Por otro lado, la relación de aspecto representa la forma del ala en función de su longitud y ancho. Este parámetro está relacionado con la ventaja aerodinámica para largos tiempos de vuelo, debido a que altos valores de relación de aspecto aumentan el coeficiente de sustentación y reducen la potencia mecánica necesaria para volar disminuyendo el arrastre (resistencia del aire) (Meseguer \& Sanz-Andrés, 2007; Pennycuick, 2008). Por ejemplo, las aves actuales que vuelan utilizando las térmicas ("soaring") usan el viento para ganar altitud, facilitado por bajos o medios valores de carga alar. En 
el caso de los albatros, además, muestran altos valores de relación de aspecto, que les permiten hacer uso del gradiente de velocidad del flujo de aire horizontal sobre el mar. En cambio, las aves con aleteo continuo generalmente muestran valores altos de carga alar. Entre estas aves, las especies que realizan vuelos cortos muestran bajos valores de relación de aspecto que las que desarrollan vuelos largos (Viscor \& Fuster, 1987; Rayner, 1988; Norberg, 2002).

Las aves muy grandes, como Pelagornis o Argentavis, no pueden alcanzar una velocidad adecuada para un vuelo de crucero de bajo costo energético, por lo que se ven obligadas a volar a niveles inferiores y a una velocidad menos eficiente (Pennycuick, 1986). Estas aves tienen mayores demandas energéticas que las pequeñas, porque la potencia disponible de sus músculos se incrementa menos con la masa corporal que la potencia requerida para el vuelo (Marden, 1994; Videler, 2005; Pennycuick, 2008). Por eso, las aves grandes tienen alas alargadas (albatros, Pelagornis) o de gran área (superficie de sustentación), como los buitres, que les permiten ganar altura utilizando las corrientes de aire ascendente y planear grandes distancias a un costo mucho menor que el aleteo (Pennycuick, 2008; Butler, 2016). La envergadura de un ave marina puede llegar a cinco veces la longitud del cuerpo y las alas tienen una mayor eficiencia aerodinámica, lo que les permite un planeo con elevación aprovechando los vientos marinos. Pero el despegue es difícil y lo realizan a la carrera o aprovechando el viento en contra (Villar et al., 2013). El vuelo planeado tiene un espectro de ángulo de planeo, que va desde vertical hacia abajo, hasta vuelos cubriendo largas distancias debido al pequeño ángulo con respecto a la horizontal como el albatros Diomedea exulans (Pennycuick, 1986). Las aves planeadoras usualmente pasan la mayor parte del tiempo planeando, obtienen la energía para el vuelo de los movimientos de la atmósfera en lugar de sus propios músculos, a esto se le llama "soaring" (Pennycuick, 1986). Es decir, aprovechan el aire para mantenerse o elevarse a más altura utilizando las corrientes térmicas que se producen por el calentamiento de la superficie terrestre y por el calentamiento de las capas de aire cercanas a este (Villar et al., 2013).

\section{MATERIALES Y MÉTODOS}

\subsection{Materiales}

Pelagornis Chilensis. Este trabajo utiliza como material a Pelagornis chilensis, MNHN SGO.PV 1061, descrito por Mayr \& Rubilar-Rogers (2010), cuyo holotipo consta de la porción dorsal del cráneo y la mayor parte del pico, once vértebras, todos los elementos de la cintura pectoral, fragmentos del esternón, de la extremidad anterior (ambos húmeros, radios y ulnas, y un carpometacarpo), de la extremidad posterior (ambos fémures, tibiotarsos y tarsometatarsos) (Fig. 2). Se encontró en la localidad El Morro, aproximadamente a $10 \mathrm{~km}$ al sur del pueblo Bahía Inglesa, desierto de Atacama, en el norte de Chile (Mayr

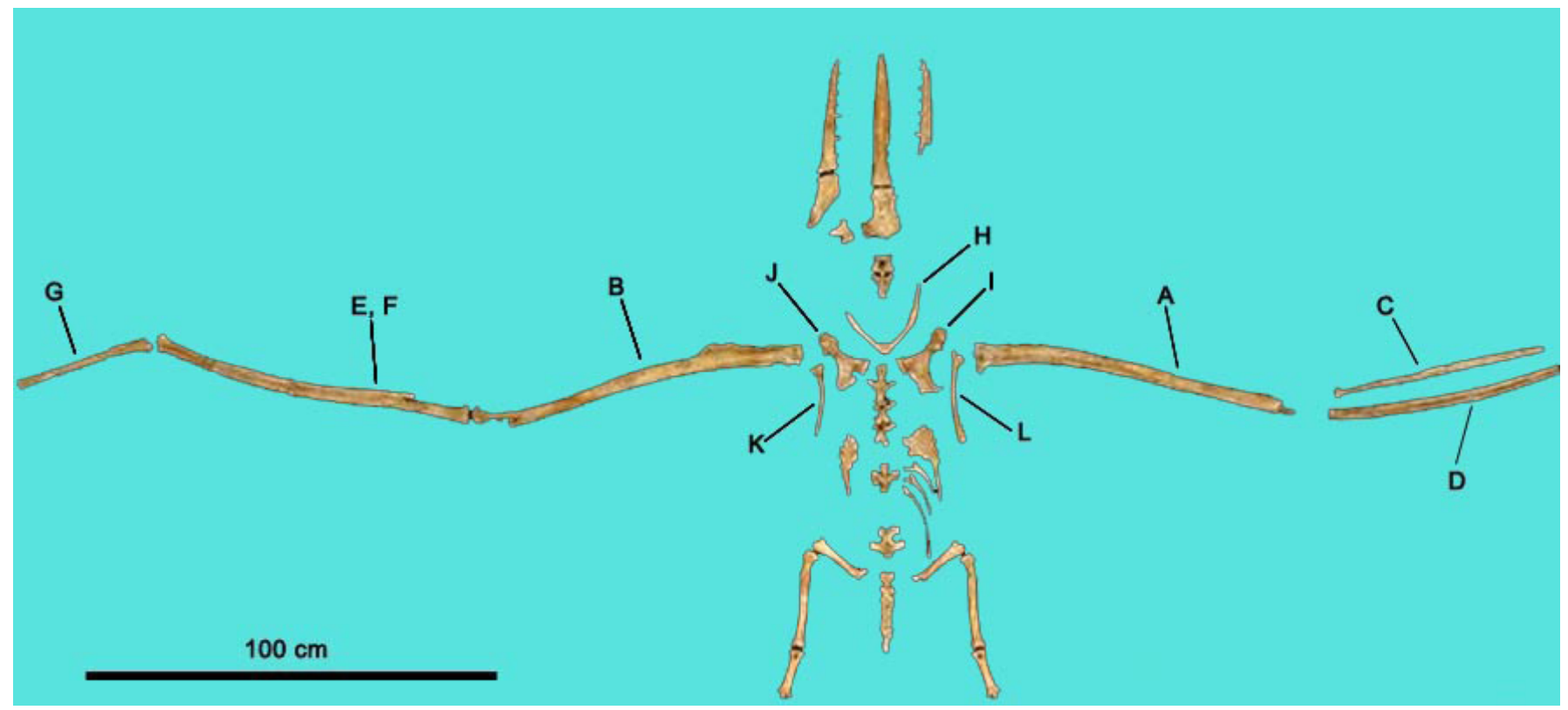

Figura 2. Pelagornis chilensis. A: húmero derecho; B: húmero izquierdo; C: radio derecho; D: ulna derecha; E, F: ulna y radio izquierdos fusionados; G: carpometacarpo izquierdo; H: fúrcula; I: coracoides derecho; J: coracoides izquierdo; K: escápula izquierda; L: escápula derecha. Imagen modificada de S. Tranker, Forschungsinstitut Senckenberg. 
\& Rubilar-Rogers, 2010), en materiales correspondientes a la Formación Bahía Inglesa, de edad Mioceno medioPlioceno temprano, alrededor de 16-4,8 millones de años (Rojo, 1985; enmendado por Marquardt et al., 2000). Se ha estimado una masa para Pelagornis chilensis de 15,6 a $28,6 \mathrm{~kg}$ y una envergadura de al menos 5,2 m (Mayr \& Rubilar-Rogers, 2010).

Thalassarche chrysostoma. Se toman los valores de Pennycuick (1982): B =2,18 m; masa $=3,79 \mathrm{~kg} ; S_{a}=0,352$ $\mathrm{m}^{2} ; \mathrm{V}_{m p}=10,9 \mathrm{~m} / \mathrm{s}(39,2 \mathrm{~km} / \mathrm{h})$ (velocidad de mínima potencia); $\mathrm{V}_{m r}=17,9 \mathrm{~m} / \mathrm{s}(64,4 \mathrm{~km} / \mathrm{h})($ velocidad con el máximo rango); $\mathrm{V}_{b g}=14 \mathrm{~m} / \mathrm{s}(50,4 \mathrm{~km} / \mathrm{h})$ (velocidad con la tasa máxima de planeo, 22). También se considera la velocidad registrada por Catry et al. (2004) en el albatros Thalassarche chrysostoma viajando en un desplazamiento de forrajeo con respecto al suelo mínima promedio de más de $110 \mathrm{~km} / \mathrm{h}$ durante aproximadamente 9 horas virtualmente sin descanso.

Diomedea exulans. Se toman los valores de Pennycuick (1982): $\mathrm{B}=3,03 \mathrm{~m} ;$ masa $=8,73 \mathrm{~kg} ; S_{a}=0,611 \mathrm{~m}^{2} ; \mathrm{V}_{m p}=$ $12,2 \mathrm{~m} / \mathrm{s}(43,9 \mathrm{~km} / \mathrm{h})$ (velocidad de mínima potencia); $\mathrm{V}_{m r}$ $=20,0 \mathrm{~m} / \mathrm{s}(72 \mathrm{~km} / \mathrm{h})$ (velocidad con el máximo rango); $\mathrm{V}_{b g}=15,7 \mathrm{~m} / \mathrm{s}(56,5 \mathrm{~km} / \mathrm{h})$ (velocidad con la tasa máxima de planeo, 23,2). En general, denotaremos la velocidad de este albatros como $v_{D}$.

\subsection{Métodos}

Para determinar los parámetros aerodinámicos se aplican relaciones alométricas entre Pelagornis chilensis y Thalassarche chrysostoma. Además, se aplican fórmulas físicas a cada especie por separado. Dada la morfología similar entre ambas aves marinas y por tener el mismo hábitat, se justifica la alometría y comparación. Para valorar la precisión del método se aplican las mismas relaciones alométricas a los albatros $T$. chrysostoma y Diomedea exulans, utilizando las velocidades observadas del primero para calcular las mismas velocidades en el segundo. Luego se comparan los valores calculados con los observados de $D$. exulans para obtener un margen de error, que será aplicado como factor de corrección a los parámetros de $P$. chilensis. No se usa ningún software o programa de simulación. Los parámetros aerodinámicos observados y las masas de los albatros se han obtenido de la literatura, así como las medidas de envergadura y estimaciones de masa de los pelagornítidos. En la literatura revisada no se han encontrado procedimientos similares. Los pasos a seguir son los siguientes:

1) Método para hallar la superficie (área) alar. Se utiliza una modificación del método del papel translucido milimetrado (o cuadrícula transparente) para medir el área del ala expuesto en Pennycuick (2008), el cual explica que al ejemplar se le extiende completamente un ala sobre la superficie de dibujo. Luego se dibuja su contorno. Se utiliza una cuadrícula (rejilla de cuadrados pequeños) rectangular transparente que se coloca sobre el trazado del ala. Se traza una línea paralela a la línea central del cuerpo, que delimita el cuerpo del ala. Se mide contando los cuadrados de la rejilla transparente. El lado del cuadrado es un dato conocido, a partir de ahí se obtiene el área de un ala que, multiplicado por dos se obtiene el área $\mathrm{S}$ del ala (Pennycuick, 2008).

La modificación del presente estudio radica en tomar la silueta a escala de un dibujo de un ave con las alas extendidas en vista dorsal, en este caso el albatros de cabeza gris (Schulenberg et al., 2010), y superponer un papel milimetrado translucido a la silueta del ave para luego contar los cuadrados y las fracciones de cuadrados que caen en el interior de toda el ala. En este trabajo vamos a asumir que ambas aves tenían una silueta similar en vista dorsal, tal como se muestra en Ksepka (2014). No obstante, esto no deja de ser una hipótesis de trabajo como una aproximación a la forma real que habría tenido Pelagornis. Entonces, se aplica el método a Pelagornis chilensis, pero con la silueta del dibujo a escala del albatros de cabeza gris del libro de aves de Schulenberg et al. (2010) y con las dimensiones del ave extinta. Teniendo el dato de la envergadura del ave y las medidas de los cuadrados, y aplicando una regla de tres simple, obtenemos el área del ala $\mathrm{S}$ del ave fósil.

2) Fuerza de sustentación de los animales voladores. Esta fuerza se genera gracias al movimiento en el aire, donde la presión en la parte superior de las alas es menor que en la inferior, con lo que se produce una fuerza ascensional o de sustentación debida a la diferencia de presiones en el fluido (Villar et al., 2013). La fuerza de sustentación, deducida de la ecuación de Bernoulli, se puede expresar como:

$$
F_{S}=\frac{1}{2} \rho S C_{S} v^{2}
$$

Esta fórmula nos indica que cuanto mayor es la velocidad mayor es la fuerza de sustentación. Del mismo modo, cuanto mayor sea el área del ala mayor será la fuerza de sustentación (Jou et al., 2009). Si se considera la resistencia, se debe tener en cuenta que alas más grandes y voluminosas generan mayor resistencia que tiende a frenar el movimiento. $\mathrm{C}_{\mathrm{s}}$ es un coeficiente de proporcionalidad que depende de la forma y estructura de las alas y del ángulo de ataque, es el coeficiente de sustentación, que en las aves es de alrededor de 0,75 (Villar et al., 2013).

Para que un cuerpo de masa $m$ no caiga, esté en equilibrio vertical, la fuerza de sustentación debe ser mayor o igual al peso, esto es:

$$
m g=\frac{1}{2} \rho S C_{s} v^{2}
$$


Donde $\mathrm{g}=10 \mathrm{~m} / \mathrm{s}^{2}$

Despejando $v$, la velocidad mínima de despegue está dada por:

$$
v \geq \sqrt{\frac{m g}{\frac{1}{2} \rho S C_{s}}}
$$

Donde el signo igual significa que el ave mantiene el vuelo y el signo mayor se aplica para el despegue (Jou et al., 2009).

La velocidad mínima de despegue $v$ también es la velocidad mínima de planeo (Villar et al., 2013). Las aves pequeñas pueden despegar con unos pocos aleteos rápidos partiendo del reposo. En cambio, las aves grandes tienen que aprovechar el impulso de la carrera, un viento rápido en contra, o se pueden lanzar desde un acantilado o un árbol alto para alcanzar la velocidad de planeo (Villar et al., 2013).

3) Alometría del vuelo, carga alar y relación de aspecto. El cambio en la forma en correlación con un cambio en el tamaño, la alometría, se define como el cambio de las proporciones en el desarrollo de un animal juvenil. Las relaciones alométricas describen cambios en la forma, que acompaña cambios en el tamaño (Kardong, 1998). La alometría se basa en comparaciones, usualmente de las diferentes partes de un animal en crecimiento; también los cambios en la relación entre las partes de diferentes especies, como los cambios evolutivos (Kardong, 1998).

En las aves, una primera relación alométrica se deriva de la ecuación de la fuerza de sustentación ya vista, de ella despejamos velocidad y obtenemos:

$$
v=\sqrt{\frac{2 F_{s}}{S C_{s} \rho}}=\sqrt{\frac{2 m g}{S C_{s} \rho}}
$$

Suponemos que g, $\rho$ y $C_{s}$ son constantes, entonces:

$$
v=\sqrt{\frac{2 m g}{S C_{s} \rho}} \alpha \sqrt{\frac{m}{S}}=>v \alpha \sqrt{\frac{m}{S}}
$$

El cociente $\mathrm{m} / \mathrm{S}$ se denomina carga del ala o carga alar (Q). La ecuación expresa que la $v$ de planeo es proporcional a la raíz cuadrada de la carga del ala (Villar et al., 2013). Se aprecia que la carga del ala cambia si el tamaño del ave varía, manteniendo todas las dimensiones en proporción. Entonces, si le cambiamos la escala al ave marina a un tamaño más grande, la masa aumenta en un factor mayor que el área del ala, de tal manera que la carga del ala aumenta (Pennycuick, 1987). Otro parámetro es la relación de aspecto $(\mathrm{R})$ :

$$
R=\frac{B^{2}}{S}
$$

Es un índice adimensional de la forma del ala, siendo alto para un ala larga y estrecha, y bajo para una corta y ancha. Una alta relación de aspecto es adecuada para un planeo eficiente, es decir, permite que el ave planee en un ángulo corto con respecto a la horizontal. (Pennycuick, 1987).

La velocidad de planeo puede expresarse en función de la longitud L del ave. Por análisis dimensional de (3) se obtienen dos relaciones:

$$
v \alpha \sqrt{L}
$$

De la ecuación (5) se observa que la velocidad de planeo es proporcional a la raíz cuadrada de la longitud del ala. La expresión también indica que a igual forma un ave más pequeña puede planear a menor velocidad que una más grande. De igual manera, de la ecuación de sustentación (2) se deduce que, a igual peso, unas alas más grandes en área, también facilitan el planeo (Villar et al., 2013). También se obtiene:

$$
v \alpha m^{\frac{1}{6}}
$$

Donde la velocidad es proporcional a la masa del ave elevada a la potencia $1 / 6$. Además, la velocidad $v$ es la velocidad mínima con la que puede planear el ave; si es menor, el peso es mayor que la fuerza de sustentación y el ave cae (Villar et al., 2013). Si tomamos en cuenta la masa, se observa de la relación que cuando aumenta la masa del ave, la velocidad de planeo debe aumentar, aunque el incremento es poco, debido al exponente $1 / 6$ (Villar et al., 2013), es decir, varía con la potencia de un sexto de la masa de las aves geométricamente similares (Pennycuick, 1987).

4) Potencia para el vuelo. La potencia que genera un ave en vuelo son dos, la potencia necesaria para la sustentación, llamada potencia inducida $P_{\text {ind }}$, y la potencia necesaria para el avance venciendo la fuerza de arrastre, llamada potencia parásita $P_{p a r}$ (Villar et al., 2013). La potencia total se expresa como la fuerza por la velocidad, es decir:

$$
P=F v
$$

En la expresión anterior para la potencia parásita, sustituimos $F$ por la fuerza de arrastre, entonces tenemos:

$$
P_{p a r}=F_{a} v=\frac{1}{2} v^{3} \rho C_{a} S_{t}
$$

De la expresión se observa que la potencia parásita crece con el cubo de la velocidad del ave. $S_{t}$ es la sección transversal a la dirección del movimiento (Villar et al., 2013).

La potencia inducida, en cambio, está en función del peso, es decir:

$$
P_{\text {ind }}=m g v_{\text {ind }}
$$


Donde $\mathrm{m}$ es la masa, $g$ es la aceleración de la gravedad y $v_{\text {ind }}$ es la velocidad inducida. Luego de sustituir $v_{\text {ind }}$ por su ecuación, la potencia inducida está dada por:

$$
P_{\text {ind }}=m g v_{\text {ind }}=\frac{4 m^{2} g^{2}}{\pi b^{2} v \rho}
$$

De la ecuación se observa que la potencia inducida varía en razón inversa a la velocidad $v$ (Villar et al., 2013). Esta ecuación se obtiene al suponer que la masa de aire que el ave impulsa hacia abajo es la que circula por un cilindro de diámetro $b$ igual a su envergadura.

La potencia total requerida para que un ave ideal pueda volar es la suma de la potencia inducida $P_{\text {ind }}$ y la potencia parásita $P_{p a r}$, donde el ave ideal posee alas sin arrastre (Pennycuick, 1975). También la potencia parásita puede expresarse alométricamente en función de la longitud del ala. De la ecuación (7) obtenemos la siguiente expresión alométrica:

$$
P_{\text {par }} \alpha L^{\frac{7}{2}}
$$

Como ambas potencias tienen las mismas unidades, la expresión alométrica de la potencia inducida es la misma (Villar et al., 2013), entonces tenemos:

$$
P_{\text {ind }} \alpha L^{\frac{7}{2}}
$$

La fuerza muscular. Esta fuerza es proporcional a $L^{2}$, entonces:

$$
F \alpha L^{2}
$$

5) Número de Reynolds. En biología, la mayoría de fenómenos se dan en condiciones de flujo laminar, pero este se mantiene hasta que la velocidad alcanza un valor determinado, que al superarlo el flujo se hace inestable y fácilmente se desestabiliza; entonces se dice que se ha desarrollado turbulencia (Jou et al., 2009). Existe una magnitud adimensional denominada número de Reynolds cuyo valor determina si un flujo es laminar o turbulento (Jou et al., 2009). Esta magnitud analiza el movimiento de los cuerpos en los fluidos a escalas espaciales diferentes (Jou et al., 2009). El número de Reynolds está definido como:

$$
R_{e}=\frac{V_{t} l \rho}{\mu}
$$

Donde $V_{t}$ es la real velocidad del fluido, $l$ es una longitud de referencia, $\rho$ es la densidad del fluido y $\mu$ es la viscosidad del fluido. En este caso el fluido es el aire. La longitud de referencia se usa para comparar dos objetos de diferente tamaño pero de forma similar. A la razón entre la viscosidad y la densidad se le llama la viscosidad cinemática, y se denota con el símbolo $v$, donde:

$$
v=\frac{\mu}{\rho}
$$

El número de Reynolds expresa la importancia de las fuerzas inerciales y viscosas. Por ejemplo, los insectos pequeños operan a bajo número de Reynolds, debido a su reducido tamaño y baja velocidad, donde las fuerzas viscosas son significativas (Pennycuick, 2008). El número de Reynolds de un ave en vuelo es del orden de 10.000 a 100.000 (Villar et al., 2013). El número de Reynolds se puede calcular para el animal en vuelo, tanto para el cuerpo como para las alas. Se diferencian en la longitud de referencia. El número de Reynolds para las alas, que utilizaremos en este trabajo, está definido como:

$$
R_{e_{\text {wing }}}=\frac{V_{t} C_{m}}{v}
$$

Donde $C_{m}$ es la medida de la cuerda del ala (área del ala, $\mathrm{S}$, dividida por la envergadura del ala, B).

$$
C_{m}=\frac{S}{B}
$$

6) Parámetros aerodinámicos de velocidad. Se tomarán distintos valores de velocidad para Thalassarche chrysostoma y para Diomedea exulans. Aparte de la velocidad mínima y la velocidad media de forrajeo asociada a una tormenta, se incluyen las siguientes:

6.1. La velocidad con el máximo rango es la mayor velocidad $V_{m r}$ definida por el punto de contacto con la curva de potencia para un ave ideal de una tangente desde el origen, donde la razón de potencia y velocidad es mínima, y por lo tanto la distancia recorrida por unidad de trabajo realizado es la máxima (Pennycuick, 1975).

6.2. La velocidad con la tasa máxima de planeo $\left(V_{b g}\right)$ se refiere a la velocidad donde la relación sustentación/ resistencia tiene el mayor valor (Pennycuick, 1982).

6.3. La velocidad de mínima potencia $\left(V_{m p}\right)$ se aprecia en la curva de potencia requerida para el vuelo en forma de $\mathrm{U}$, que es la suma de la potencia inducida y la potencia parásita, donde alcanza un mínimo a la velocidad $V_{m p}$, en la cual la potencia total requerida es la potencia mínima absoluta $P_{a m}$ (Pennycuick, 1982; Villar et al., 2013).

Las velocidades $V_{m p}$ y $V_{b g}$ en el vuelo batido o de aleteo se pueden usar para estimar cómo estas deberían variar en diferentes tamaños de Procellariiformes, es decir, que para aves geométricamente similares, la velocidad para el rango máximo debe variar de la misma manera que las velocidades de planeo, pero con la sexta potencia de la masa (Pennycuick, 1987). 
Estas velocidades tienen sus respectivas fórmulas, pero dada su complejidad no serán presentadas en este trabajo. Pueden ser consultadas en Pennycuick (1975).

7) Alometría aplicada a Diomedea exulans. Para definir qué camino de alometría define mejor la velocidad de Pelagornis chilensis, se utiliza otro albatros, Diomedea exulans, del que se tienen sus parámetros de vuelo (Pennycuick, 1982). Para ello, se le relaciona alométricamente con Thalassarche chrysostoma y se obtienen valores de velocidad. La diferencia entre los valores observados y los calculados de $D$. exulans nos dará un porcentaje de error, que se utiliza para ajustar los resultados de $P$. chilensis.

\section{RESULTADOS}

La aplicación de las fórmulas nos arroja valores de parámetros aerodinámicos para las especies en estudio, cuyo desarrollo matemático se expone en el Apéndice.

\subsection{Velocidades de Pelagornis chilensis}

P. chilensis

T. chrysostoma

(con masa promedio)

$$
v_{p}=48,9 \frac{\mathrm{km}}{\mathrm{h}} \quad v_{a}=38,9 \frac{\mathrm{km}}{\mathrm{h}}
$$

Tabla 1: Velocidades mínimas de $P$. chilensis y $T$. chrysostoma por fórmula de sustentación (2). (Ver Apéndice, puntos 1 y 2$)$.

Velocidad media real de forrajeo asociada a una tormenta en la Antártida

Velocidad de mínima potencia

$$
v_{p} \geq 196,7 \frac{\mathrm{km}}{\mathrm{h}} \quad v_{m p}=60,7 \frac{\mathrm{km}}{\mathrm{h}}
$$

Velocidad con el máximo Velocidad con la tasa rango máxima de planeo

$$
v_{m r}=99,8 \frac{\mathrm{km}}{\mathrm{h}} \quad v_{b g}=78,1 \frac{\mathrm{km}}{\mathrm{h}}
$$

Tabla 2: Velocidades de $P$. chilensis por alometría con $T$. chrysostoma en función de la longitud $L$ del ave, según fórmula (5). (Ver Apéndice, punto 3).
T. chrysostoma P. chilensis Incremento (\%)

\begin{tabular}{lccc}
\hline v forrajeo tormenta & 127 & 196,7 & 54,9 \\
v max rango & 64,4 & 99,8 & 54,9 \\
v tasa max planeo & 50,4 & 78,1 & 54,9 \\
v min potencia & 39,2 & 60,7 & 54,8 \\
\hline
\end{tabular}

Tabla 3: Comparación de velocidades $(\mathrm{km} / \mathrm{h})$, calculadas de $P$. chilensis de Tabla 2 con las observadas del albatros.

Velocidades $\mathrm{v}$ mínima $\mathrm{v}$ mínima $\mathrm{v}$ tasa $\quad \mathrm{v}$ máxima $\mathrm{v}$ forrajeo en albatros fórmula potencia máxima rango tormenta planeo

\begin{tabular}{lccccc}
$\mathrm{kg}$ & $38,9 \mathrm{~km} / \mathrm{h}$ & $39,2 \mathrm{~km} / \mathrm{h}$ & $50,4 \mathrm{~km} / \mathrm{h}$ & $64,4 \mathrm{~km} / \mathrm{h}$ & $127 \mathrm{~km} / \mathrm{h}$ \\
\hline 16 & 49,4 & 49,8 & 64 & 81,8 & 161,3 \\
$\begin{array}{l}22,5 \\
\text { (media) }\end{array}$ & 52,5 & 52,9 & 68 & 86,9 & 171,5 \\
29 & 54,5 & 54,9 & 70,6 & 90,2 & 177,8
\end{tabular}

Tabla 4: Velocidades de $P$. chilensis por alometría con $T$. chrysostoma en función de la masa y para diferentes velocidades de T. chrysostoma, según fórmula (6). (Ver Apéndice, punto 4).

\section{T. chrysostoma P. chilensis Incremento (\%)}

\begin{tabular}{lrrr}
\hline v min potencia & 39,2 & 60,7 & 54,8 \\
v min fórmula & 38,9 & $41-55,4$ & $5,4-42,4$
\end{tabular}

Tabla 5: Comparación de las velocidades $(\mathrm{km} / \mathrm{h})$ de mínima potencia de ambas aves, calculada por alometría en el caso del ave extinta y observada en el albatros; y las velocidades de vuelo mínimas calculadas por la fórmula de sustentación (2), en el caso de Pelagornis con la masa mínima y máxima estimada.

\subsection{Velocidades de Diomedea exulans}

Velocidad con la mínima potencia máximo rango tasa máxima de planeo

$$
v_{D}=46,2 \frac{\mathrm{km}}{\mathrm{h}} \quad v_{D}=75,9 \frac{\mathrm{km}}{\mathrm{h}} \quad v_{D}=59,4 \frac{\mathrm{km}}{\mathrm{h}}
$$

Tabla 6: Velocidades de Diomedea exulans por alometría con T. chrysostoma en función de la longitud $L$ del ave, según fórmula (5). (Ver Apéndice, punto 7.1). 


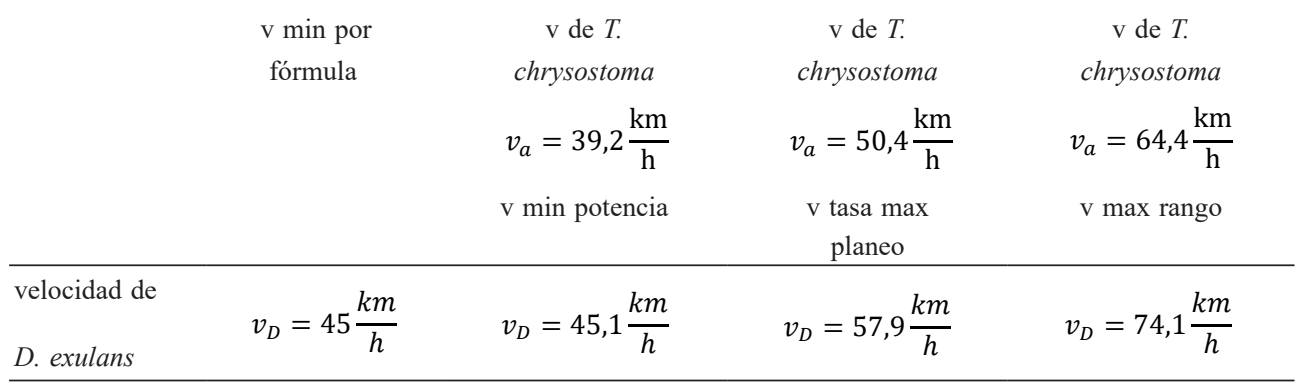

Tabla 7: Velocidades de D. exulans, por fórmula de sustentación (2) y por alometría, con $T$. chrysostoma en función de la masa, según fórmula (6). (Ver Apéndice, puntos 7.2 y 8).

\subsection{Otros parámetros aerodinámicos: potencia parásita e inducida, fuerza muscular y modelo de flujo}

Potencia parásita Potencia inducida Fuerza muscular

$\begin{array}{ccc} & P_{\text {par }}=21,1 & P_{\text {ind }}=21,1 \\ \text { veces la de } T . & \text { veces la de } T . & F=5,7 \text { veces la de } T . \\ \text { chrysilensis } & \text { chrostoma } & \text { chrysostoma }\end{array}$

Tabla 8: Potencia parásita e inducida y fuerza muscular de $P$. chilensis por alometría con T. chrysostoma, según fórmulas (10), (11) y (12). (Ver Apéndice, punto 5).

\begin{tabular}{lcccc} 
& $\begin{array}{c}\mathrm{v} \text { min } \\
\text { potencia }\end{array}$ & $\begin{array}{c}\mathrm{v} \text { tasa max } \\
\text { planeo }\end{array}$ & $\begin{array}{c}\mathrm{v} \max \\
\text { rango }\end{array}$ & $\begin{array}{c}\mathrm{v} \text { forrajeo } \\
\text { tormenta }\end{array}$ \\
\hline P. chilensis & 52,9 & 68 & 86,9 & 171,5 \\
T. chrysostoma & 39,2 & 50,4 & 64,4 & 127 \\
$P_{\text {par }} / P_{\text {par }}^{\prime}$ & 14 & 14 & 14 & 14 \\
\hline
\end{tabular}

Tabla 9: Razón potencia parásita $P$. chilensis/potencia parásita T. chrysostoma para diferentes velocidades, observadas para el albatros y calculadas por alometría en función de la masa para el pelagornítido. (Ver Apéndice, punto 9).

\begin{tabular}{lcccc} 
& $\begin{array}{c}\mathrm{v} \text { min } \\
\text { potencia }\end{array}$ & $\begin{array}{c}\mathrm{v} \text { tasa max } \\
\text { planeo }\end{array}$ & $\begin{array}{c}\mathrm{v} \max \\
\text { rango }\end{array}$ & $\begin{array}{c}\mathrm{v} \text { forrajeo } \\
\text { tormenta }\end{array}$ \\
\hline P. chilensis & 60,7 & 78,1 & 99,8 & 196,7 \\
T. chrysostoma & 39,2 & 50,4 & 64,4 & 127 \\
$P_{\text {par }} / P_{\text {par }}^{\prime}$ & 21,2 & 21,3 & 21,3 & 21,2 \\
\hline
\end{tabular}

Tabla 10: Razón potencia parásita de ambas aves utilizando la velocidad calculada para el ave extinta aplicando alometría en función de la longitud L del ave y la velocidad observada del albatros.

\begin{tabular}{|c|c|c|c|c|}
\hline & $\begin{array}{l}\text { Con velocidad } \\
\text { media de } \\
\text { forrajeo de } \\
\text { albatros } \\
\text { asociada a } \\
\text { tormenta: }\end{array}$ & $\begin{array}{l}\text { Con } \\
\text { velocidad } \\
\text { calculada } \\
\text { asociada a } \\
\text { tormenta: }\end{array}$ & $\begin{array}{l}\text { Con velocidad } \\
\text { de albatros } \\
\text { con la mínima } \\
\text { potencia: } \\
\\
y=392 \mathrm{~km}\end{array}$ & $\begin{array}{l}\text { Con } \\
\text { velocidad } \\
\text { calculada con } \\
\text { la mínima } \\
\text { potencia: }\end{array}$ \\
\hline & $v=127 \frac{\mathrm{km}}{\mathrm{h}}$ & $v=167 \frac{\mathrm{km}}{\mathrm{h}}$ & & $v=50,5 \frac{\mathrm{km}}{\mathrm{h}}$ \\
\hline P. chilensis & 611931 & 804351 & 188953 & 242692 \\
\hline T. chrysostoma & 386314 & & 119287 & \\
\hline
\end{tabular}

Tabla 11: Modelos de flujo para el ala de ambas aves, en valores

de Re. (Ver Apéndice, punto 6).

P. chilensis D. exulans T. chrysostoma

\begin{tabular}{llll}
\hline Carga alar & $16,9 \mathrm{~kg} / \mathrm{m}^{2}$ & $14,29 \mathrm{~kg} / \mathrm{m}^{2}$ & $10,77 \mathrm{~kg} / \mathrm{m}^{2}$ \\
Relación de aspecto & 20,33 & 15,03 & 13,5 \\
\hline
\end{tabular}

Tabla 12: Valores de carga alar y relación de aspecto del pelagornítido y los albatros.

\section{DISCUSIÓN}

\subsection{Velocidad de Pelagornis chilensis}

Utilizando la fórmula de la fuerza de sustentación despejamos la velocidad mínima de vuelo, tanto para el pelagornítido como para el albatros de cabeza gris (Tabla 1). Estos resultados teóricos consideran un vuelo planeado y sin turbulencia. Se aprecia que el pelagornítido tiene una velocidad mínima calculada (Ver Apéndice, punto 1) de $48,9 \mathrm{~km} / \mathrm{h}$ con $22,5 \mathrm{~kg}$ de peso promedio, mientras que el albatros tiene $38,9 \mathrm{~km} / \mathrm{h}$. Esta última velocidad calculada coincide con la velocidad de mínima potencia de vuelo del albatros de cabeza gris registrada (observada) por Pennycuick (1982), dada por: $V_{m p}=10,9 \mathrm{~m} / \mathrm{s} \quad(39,2$ $\mathrm{km} / \mathrm{h}$ ). Pero la velocidad mínima no es la velocidad de 
mínima potencia; la primera es menor, como se aprecia en la figura 14.26 en Villar et al. (2013), lo que revelaría una desviación con respecto al valor real de velocidad mínima con el uso de la fórmula.

Catry et al. (2004) registró una velocidad mínima promedio con respecto al suelo para un albatros de cabeza gris de más de $110 \mathrm{~km} / \mathrm{h}$ durante 9 horas y sin descanso, lo que representa más del doble de la calculada. Esta diferencia se debe a que la velocidad mínima promedio registrada para el albatros no es la velocidad mínima de vuelo de las aves; esta se refiere a que si la velocidad es menor el ave cae, mientras que la otra es el promedio de registrar velocidades en varias locaciones, donde en cada una se registró una velocidad mínima y una máxima, y se promediaron las mínimas obteniéndose una velocidad promedio mayor a $110 \mathrm{~km} / \mathrm{h}$ (Catry et al., 2004). Estas velocidades se dieron cuando el ave estaba en vuelo de forrajeo, donde las velocidades son mayores que en un vuelo de crucero, $y$, lo más importante, con la influencia de vientos de tormenta, que favorecieron al desarrollo de gran velocidad del albatros.

Se observa un incremento igual en todos los casos (Tabla 3) debido a que es la misma relación alométrica para los cuatro casos. Pero, por ejemplo, al comparar las velocidades de mínima potencia de ambas aves, calculada por alometría en el caso del ave extinta, y las velocidades de vuelo mínimas calculadas por la fórmula de sustentación (segunda fila de la Tabla 5), encontramos una relevante diferencia en el incremento. Esta diferencia en el incremento entre el ave actual y el ave fósil, podría deberse a la aplicación de metodologías diferentes: fórmula y alometría.

Aplicando alometría de la velocidad en función de la masa, obtenemos tres relaciones alométricas, para 16, 22,5 (media) y $29 \mathrm{~kg}$ de peso de Pelagornis, en las cuales reemplazamos cinco velocidades del albatros: una calculada y cuatro registradas en diferentes condiciones de vuelo (Tabla 4). Observamos en la Tabla 4 que la velocidad calculada del pelagornítido tiene un amplio rango, al igual que el albatros, desde un valor mínimo de $52,5 \mathrm{~km} / \mathrm{h}$ calculada por fórmula y con la media del peso $(22,5 \mathrm{~kg})$, hasta un máximo de $171,5 \mathrm{~km} / \mathrm{h}$ en condiciones de forrajeo y con vientos de tormenta que facilitan el desarrollo de grandes velocidades. Sin embargo, aplicando alometría en función de la longitud $L$, el rango de velocidades del pelagorntído varía (Tabla 3), aumentado los valores mínimos y máximos, 60,7 y $196,7 \mathrm{~km} / \mathrm{h}$, para las mismas condiciones de vuelo. En la Tabla 4 se puede observar que el incremento entre las velocidades del ave extinta, con el peso promedio de $22,5 \mathrm{~kg}$, con respecto a las correspondientes velocidades del albatros es $35 \%$, a diferencia del 54,9\% de la Tabla 3, donde se aplicó alometría en función de la longitud $L$.

\subsection{Razón potencia parásita $P$. chilensis/T. chrysostoma}

Se observa en los resultados, en la sección otros parámetros aerodinámicos (Tabla 8), que el pelagornítido, por alometría en función a $L$, necesita una potencia parásita y una potencia inducida de 21,1 veces la del albatros de cabeza gris. Es decir, la potencia necesaria para el avance venciendo la fuerza de arrastre y la potencia para la sustentación usando para ello las fórmulas (10) y (11) respectivamente. Asimismo, por alometría en función a $L$ (fórmula (12)), la fuerza muscular del pelagornítido es 5,7 veces la del albatros de cabeza gris. Comparamos esos resultados con otro valor de la razón potencia parásita pelagornítido/potencia parásita albatros usando la fórmula no alométrica de la potencia parásita (7), cuyo desarrollo matemático es propio del autor y se muestra en el Apéndice, punto 9. De este procedimiento, se obtiene el siguiente valor:

$$
\frac{P_{\text {par }}}{P_{\text {par }}^{\prime}}=14
$$

Lo que significa que la potencia parásita del pelagornítido es 14 veces la potencia parásita del albatros. Se usó para los cálculos las velocidades de mínima potencia, la observada para T. chrysostoma y la calculada por alometría en función de la masa con la media del peso para $P$. chilensis (Tabla 4). La razón potencia parásita de ambas aves tienen el mismo valor para las diferentes velocidades calculadas por alometría en función de la masa del ave extinta y las observadas del albatros, como se observa en la Tabla 9.

Usando el mismo procedimiento (Apéndice, punto 9), vamos a comparar la razón potencia parásita de ambas aves utilizando la velocidad calculada para Pelagornis aplicando alometría en función de la longitud $L$ del ave, resultados que se pueden observar en la Tabla 10. De las Tablas 9 y 10 , se observa que los valores de la razón de la potencia parásita entre las aves varían significativamente $(52 \%$ de incremento) entre usar alometría de la velocidad en función de la masa y alometría en función de la longitud $\mathrm{L}$ del ave extinta, 14 y 21,25 respectivamente.

Se observa en la Tabla 8 que el resultado de 21,1 obtenido con la fórmula alométrica de la potencia parásita (10), es casi igual al resultado, en promedio 21,25 (Tabla 10), obtenido por la fórmula parásita (7) en función de la velocidad y de una dimensión lineal $r$ (ver Apéndice, punto 9) alimentada con los valores de velocidad del Pelagornis hallados por alometría en función de la longitud $L$ del ave. No ocurre lo mismo con los resultados de potencia parásita obtenidos con las velocidades del pelagornítido halladas aplicando alometría a la velocidad en función de la masa (Tabla 9). De los cálculos hechos, saldrían fortalecidas las velocidades por alometría en función a $\mathrm{L}$ de la Tabla 10 ; 
pero hay que considerar que en este caso, tanto la fórmula alométrica (10) de la potencia parásita y las velocidades halladas por alometría del ave fósil y reemplazadas en la fórmula de la razón potencia parásita $\left(\frac{P_{p a r}}{P_{\text {par }}^{\prime}}\right.$, ver Apéndice, punto 9) están en función de $L$. En ambos casos, esta dependencia de una sola variable le daría coherencia a los resultados en valores muy próximos, 21,1 y 21,25. Sin embargo, no se puede afirmar que dichas velocidades necesariamente reflejarían mejor las velocidades del ave fósil.

\subsection{Velocidad calculada y velocidad observada de Diomedea exulans}

En la siguiente tabla vemos los valores calculados y observados para el albatros Diomedea exulans.

\begin{tabular}{lllllll} 
& $\begin{array}{l}\mathrm{v} \text { min } \\
\text { potencia }\end{array}$ & $\begin{array}{l}\Delta \\
\%\end{array}$ & $\begin{array}{l}\mathrm{v} \text { max tasa } \\
\text { planeo }\end{array}$ & $\begin{array}{l}\Delta \\
\%\end{array}$ & $\begin{array}{l}\mathrm{v} \max \\
\text { rango }\end{array}$ & $\begin{array}{c}\Delta \\
\%\end{array}$ \\
\hline Alometría con L & 46,2 & 5,2 & 59,4 & 5,1 & 75,9 & 5,4 \\
Alometría con m & 45,1 & 2,7 & 57,9 & 2,5 & 74,1 & 2,9 \\
Observado & 43,9 & & 56,5 & & 72 & \\
\hline
\end{tabular}

Tabla 13: Valores observados de $D$. exulans para diferentes velocidades vs valores calculados de las mismas velocidades por alometría con respecto a $T$. chrysostoma en función de la longitud L y la masa m. (Ver Apéndice, puntos 7.1 y 7.2).

En la Tabla 13 se observa que los valores obtenidos por alometría en función de la masa (ver Apéndice, punto 7.2) se acercan más a los valores reales de las velocidades del albatros Diomedea exulans, que los valores obtenidos por alometría en función de la longitud (ver Apéndice, punto 7.1). El porcentaje promedio de incremento de la velocidad con alometría con L (longitud) es 5,23\%, mientras que el incremento de la velocidad con alometría con m (masa) es $2,7 \%$. Por lo tanto, siendo cerca del doble el porcentaje de incremento de alometría en función de la longitud con respecto a los valores obtenidos por alometría en función de la masa, se puede afirmar que a través de esta última alometría se obtienen valores más cercanos a los valores reales de velocidad del albatros $D$. exulans.

\subsection{El factor de corrección para las velocidades de Pelagornis chilensis}

La aplicación de alometrías en el albatros actual $D$. exulans, nos lleva a plantear las siguientes afirmaciones: 1) los resultados de velocidades calculadas de Pelagornis chilensis con alometría en función de la masa son más cercanos a los valores que habría tenido el ave fósil realmente. 2) El porcentaje de incremento de 2,7\% en alometría en función de la masa con respecto a los valores reales del albatros, se usará como factor de corrección para ajustar en $-2,7 \%$ las cifras calculadas del ave fósil y acercarlas más a los valores que habría tenido realmente.

Los valores ajustados (corregidos) de las velocidades de $P$. chilensis por alometría en función de la masa quedarían de la siguiente forma (Tabla 14):

\begin{tabular}{lccccc}
$\begin{array}{l}\text { Velocidades } \\
\text { albatros }\end{array}$ & $\mathrm{v}$ min formula & $\begin{array}{l}\mathrm{v} \min \\
\text { potencia }\end{array}$ & $\begin{array}{l}\mathrm{v} \text { tasa max } \\
\text { planeo }\end{array}$ & $\begin{array}{l}\mathrm{v} \max \\
\text { rango }\end{array}$ & $\begin{array}{l}\mathrm{v} \text { forrajeo } \\
\text { tormenta }\end{array}$ \\
$\mathrm{kg}$ & $\begin{array}{l}38,9 \mathrm{~km} / \mathrm{h} \\
\mathrm{kg}\end{array}$ & $39,2 \mathrm{~km} / \mathrm{h}$ & $50,4 \mathrm{~km} / \mathrm{h}$ & $64,4 \mathrm{~km} / \mathrm{h}$ & $127 \mathrm{~km} / \mathrm{h}$ \\
\hline $\begin{array}{l}22,5 \text { (media) } \\
52,5\end{array}$ & 52,9 & 68 & 86,9 & 171,5 \\
$\begin{array}{l}\text { corregido y } \\
\text { redondeado }\end{array}$ & 51 & 51 & 66 & 85 & 167 \\
\hline
\end{tabular}

Tabla 14: Valores ajustados y redondeados de las velocidades de $P$. chilensis para los obtenidos por alometría con respecto a $T$. chrysostoma en función de la masa.

\subsection{La velocidad mínima de Pelagornis chilensis}

La velocidad mínima de Pelagornis, calculada con la fórmula de sustentación, arrojaría un valor por encima del valor real, como se deduce indirectamente de Villar et al. (2013). Esta diferencia se debería a que el fluido en la ecuación de Bernoulli, en este caso el aire, es considerado un fluido ideal de flujo estacionario, es decir, sin viscosidad, a temperatura constante, a presión constante y densidad constante. Además, se espera que la estela turbulenta que aparece detrás del ala sea relativamente débil, por lo que la sustentación no cambia, debido a que no hay cambios de presión bruscos producidos por los torbellinos (Villar et al., 2013). La diferencia entre la velocidad mínima real y la mínima calculada del ave fósil, se deduce de la figura 14.26, b, en Villar et al. (2013). En esta gráfica se muestra la potencia necesaria para el vuelo de un ave de $400 \mathrm{~g}$, y de ella se deduce que el ave puede volar a velocidades entre 22 y $66 \mathrm{~km} / \mathrm{h}$ y consume la potencia mínima a una velocidad de $42 \mathrm{~km} / \mathrm{h}$. Además, se hace hincapié que para iniciar el vuelo y alcanzar la velocidad mínima de 22 $\mathrm{km} / \mathrm{h}$ debe utilizar técnicas diferentes de vuelo y recursos de energía adicionales de corta duración. En este caso, la velocidad mínima de esta ave de 400 g está cerca del valor de la mitad de la velocidad de mínima potencia. Sin embargo, en Pennycuick (1987) se afirma que la velocidad de planeo mínima estimada oscila de alrededor de $4 \mathrm{~m} / \mathrm{s}$ en petreles pequeños hasta aproximadamente $12 \mathrm{~m} / \mathrm{s}$ (43 $\mathrm{km} / \mathrm{h}$ ) en albatros grandes, valor mayor que la velocidad mínima calculada por fórmula de $39 \mathrm{~km} / \mathrm{h}$ para el albatros 
de cabeza gris, Thalassarche chrysostoma, que no es el albatros más grande, y por lo tanto es coherente que tenga una velocidad menor. El caso expuesto en Villar et al. (2013) podría no ser aplicable al albatros de cabeza gris ni a Pelagornis chilensis, porque se trata de un ave pequeña; $\mathrm{y}$, más bien, según Pennycuick (1987) la velocidad de planeo mínima en aves marinas grandes sería similar a la velocidad de mínima potencia de las mismas; entonces, no se descarta la velocidad mínima calculada por la fórmula.

\subsection{Razón potencia parásita ave extinta / ave actual con factor de corrección}

Tomando las velocidades calculadas por alometría en función de la masa y corregidas, se calculará de nuevo la razón potencia parásita entre las aves. Se obtiene una media de 12,75 , es decir, $P$. chilensis tiene una potencia parásita promedio de 12,75 veces la potencia parasita del albatros de cabeza gris, como se puede ver en la Tabla 15.

\begin{tabular}{lcccc} 
& $\begin{array}{c}\mathrm{V} \text { min } \\
\text { potencia }\end{array}$ & $\begin{array}{c}\mathrm{v} \text { tasa max } \\
\text { planeo }\end{array}$ & $\begin{array}{c}\mathrm{v} \text { rango } \\
\max \end{array}$ & $\begin{array}{c}\mathrm{v} \text { forrajeo } \\
\text { tormenta }\end{array}$ \\
\hline P. chilensis & 51 & 66 & 85 & 167 \\
T. chrysostoma & 39,2 & 50,4 & 64,4 & 127 \\
$P_{\text {par }} / P_{\text {par }}^{\prime}$ & 12,5 & 12,8 & 13,1 & 12.9 \\
\hline
\end{tabular}

Tabla 15: Razón potencia parásita entre ambas aves para las distintas velocidades calculadas y corregidas por alometría en función de la masa para $P$. chilensis, mostradas en la Tabla 14, y las velocidades observadas para T. chrysostoma.

\subsection{Modelo de flujo de Pelagornis chilensis}

En cuanto al modelo de flujo, se puede apreciar (Tabla 11) que a una misma velocidad, en este caso la de forrajeo con tormenta y la de mínima potencia del albatros, el $R_{e}$ para el ala del pelagornítido es 1,58 veces el $R_{e_{w i n g}}$ del albatros para ambos casos, lo que indica una mayor turbulencia sobre las alas del ave fósil, produciendo una mayor resistencia al avance.

Comparando las velocidades máximas del albatros y del pelagornítido utilizadas en este trabajo, 127 y $167 \mathrm{~km} / \mathrm{h}$ respectivamente, el $R_{e_{\text {wing }}}$ del ave fósil es 2,1 veces el valor del $R_{e_{\text {wing }}}$ del ave actual; esto nos indica que el pelagornítido está sometido a una mayor resistencia al avance que el albatros; por lo tanto requiere de mayor gasto energético llegar a esa velocidad de forrajeo con tormenta. Para tener una referencia que nos grafique lo significativo del valor del número de Reynolds con respecto a otro, tomaremos las
10 veces que hay entre el $R_{e}$ de un pato volando (300.000) y el $R_{e}$ de una gran libélula (30.000) (Vogel, 1996).

\subsection{Parámetros de vuelo de Pelagornis sandersi y de $P$. chilensis}

A diferencia de esta investigación que prescinde de un software de vuelo, el trabajo de Ksepka (2014) estudia el vuelo de Pelagornis sandersi con un modelado por computadora, utilizando el software Flight 1.25. Ksepka (2014) justifica el uso del programa de computadora argumentando que el grupo Pelagornithidae evolucionó en morfologías del esqueleto altamente modificadas y que no sería plausible hacer inferencias significativas sobre su estilo de vuelo y ecología basadas en parientes actuales. Por lo tanto, el modelado por computadora proporciona el mejor camino hacia la comprensión de las capacidades de vuelo de estas aves fósiles (Ksepka, 2014). Flight 1.25 modela vuelo de aves en condiciones especificadas por el usuario, modela tanto el aleteo como el planeo (Ksepka, 2014). En el trabajo de Ksepka (2014) se aplica para deducir parámetros de vuelo de Pelagornis sandersi, pero el objetivo no es hallar velocidades de vuelo, como sí lo es en este trabajo con Pelagornis chilensis.

Ksepka (2014) no calcula ninguna velocidad del Pelagornis sandersi, lo que encuentra con Flight 1.25 es una relación significativa entre la sustentación y la resistencia (arrastre) para un determinado rango de velocidad; específicamente, predice que a velocidades entre 10,6 y $17,0 \mathrm{~m} / \mathrm{s}$ la razón sustentación/resistencia alcanza altos valores, entre 21,0 a 23,9 en vuelo planeado, $\mathrm{y}$, además, pudo planear a altas velocidades con bajas tasas de descenso. Pennycuick (1982) determinó para el albatros de cabeza gris una velocidad de $14 \mathrm{~m} / \mathrm{s}$ para una máxima tasa de planeo (razón sustentación/resistencia) de 22, y para el albatros Diomedea exulans una velocidad de 15,7 $\mathrm{m} / \mathrm{s}$ para una máxima tasa de 23,2 ; cifras que están dentro de los rangos calculados para Pelagornis sandersi, lo que reflejaría una eficiencia y modo de vuelo del ave fósil similar a los albatros mencionados. Estas coincidencias de estos parámetros de vuelo fortalecen la justificación de la comparación del vuelo del ave extinta con el de los albatros actuales. Se aprecia en la figura 2-B de Ksepka (2014) los altos valores de la tasa sustentación/resistencia para el rango de velocidad especificado; pero también velocidades cercanas a $\operatorname{los} 40 \mathrm{~m} / \mathrm{s}(144 \mathrm{~km} / \mathrm{h})$, que equivale a valores cercanos a 12 para la tasa estudiada; lo que significa que a mayores velocidades la resistencia va aumentando y el ave necesita gastar más energía para mantener el vuelo o incrementar la velocidad. Entonces, según el gráfico de Ksepka (2014), Pelagornis sandersi podía alcanzar velocidades mayores a $140 \mathrm{~km} / \mathrm{h}$, lo que no significa que esta sea una velocidad máxima, porque el programa Flight 1.25 no fue utilizado para hallar velocidades. Este 
gráfico y las velocidades máximas mostradas en el eje $\mathrm{X}$ son coherentes con la velocidad máxima calculada para Pelagornis chilensis. Además, con el modelado de los parámetros de vuelo, Ksepka (2014) dedujo que Pelagornis sandersi fue capaz de vuelos planeados altamente eficientes y que habría explotado la estrategia de largo alcance de utilizar las corrientes de aire ascendentes ("soaring") similar a los albatros actuales.

Ksepka (2014), Serrano \& Chiappe (2017) y Serrano et al. $(2017,2018)$ han utilizado otras herramientas metodológicas para estudiar el vuelo de aves del Cretácico, como el modelado computacional, análisis morfofuncional, análisis multivariado de elementos de extremidades anteriores y modelos de regresión. Asimismo, Cannell (2020) utiliza el software Flight 1.25 para estudiar las limitaciones de vuelo de aves gigantes del Mioceno; pero ninguno aplica alometría ni determina velocidad de vuelo de un ave extinta como el presente trabajo.

\subsection{El vuelo del albatros y los Pelagornithidae}

Como ya se ha visto morfológicamente (Ksepka, 2014) el tipo de ala del pelagornítido es similar a la del albatros, es decir el ala de un ave marina pelágica de tipo ala de veleo en el mar; esta desarrolla un planeo activo en alta mar o veleo. En Pennycuick (1982) se distinguieron tres tipos de vuelo para petreles y albatros (procellariiformes), observados en South Georgia y vecindades, estos eran: el planeo, el planeo-aleteo y el aleteo. A las aves más pequeñas nunca se les vio planeando sobre el mar, mientras que a las cinco especies grandes (cuatro albatros y el petrel gigante) infrecuentemente se les vio en aleteo o planeoaleteo sobre el mar, sobre todo se les observó con este tipo de vuelo en condiciones de calma (viento inferior a $3 \mathrm{~m} / \mathrm{s}$ ); pero cuando hubo un viento apreciable dejaron el aleteo por el planeo para poder aprovechar el viento sobre el mar (Pennycuick, 1982). En el caso exclusivo de los albatros, nunca baten sus alas, excepto en vientos muy leves (y, a veces, ni siquiera entonces), por lo que pueden ser representados como pasando todo su tiempo en vuelo planeado (Pennycuick, 1982). Esta característica de vuelo de los albatros está directamente relacionada a altos valores de la carga alar, que les permite desarrollar mayor velocidad en fases de planeo; pero, en contraste, les da menos maniobrabilidad. Observamos (redondeando cifras de la Tabla 12) que Pelagornis chilenses tiene una carga alar de casi 17, mientras que Diomedea exulans 14,3 y Thalassarche chrysostoma 13,5; lo que es compatible con la mayor velocidad que desarrolla el ave extinta sobre las aves actuales propuesta en este estudio; sin embargo, le trae la desventaja de una menor maniobrabilidad de vuelo.

Pennycuick (1982) observó el vuelo de los Procellariiformes ante la presencia de viento de cola y de cabeza. En el caso de las aves pequeñas, su velocidad maximiza la relación distancia recorrida-combustible consumido, mientras que los albatros y petreles gigantes volaban principalmente en modo planeo y cuando volaban contra el viento seguían un recorrido en zigzag aprovechando la elevación de la pendiente. Al parecer, la elevación de la pendiente era la fuente de energía primaria para las especies grandes y volaban a velocidades relativamente bajas para hacer uso de este efecto, mientras que la mayor parte del requerimiento de energía de las aves pequeñas la sacaban a partir de la potencia muscular y muy infrecuentemente de la elevación de la pendiente. Esto supone que las aves grandes gastan menos energía de su metabolismo basal que las aves pequeñas (Pennycuick, 1982). Además, el investigador ha comprobado que, aunque las velocidades absolutas son altas en las especies grandes, estas aves vuelan lentamente en relación con sus velocidades de potencia mínimas, es decir, están volando a bajas velocidades relativas. La afirmación de Pennycuick (1982) sobre el requerimiento de energía para las aves grandes alcanza a los Pelagornithidae. La elevación de la pendiente ("lift slope") es el coeficiente de sustentación $C_{L}$ dividido entre el ángulo de ataque $\varphi$; es decir, es la variación del coeficiente de sustentación en función del ángulo de ataque del ala, que se visualiza en las coordenadas cartesianas como una pendiente, donde el eje $y$ es $\mathrm{C}_{\mathrm{L}}$ y el eje $x$ es $\varphi$ (Anderson, 2001).

Las observaciones actuales sugieren que los albatros y los petreles más grandes extraen energía de la gradiente vertical de la velocidad del viento en las subidas contra el viento y en las bajadas del viento; además, el gradiente de viento puede suministrar relativamente más energía en vuelo a favor del viento, pero no se obtuvieron observaciones directas para respaldar esto (Pennycuick, 1982). El aprovechamiento de la gradiente de velocidad del viento horizontal sobre el mar está relacionado con un mayor valor de la relación de aspecto, para las tres especies de este trabajo Pelagornis chilensis posee una relación de aspecto de 20,3 contra 15 de Diomedea exulans y 13,5 de Thalassarche chrysostoma (ver Tabla 12). Este valor le da al ave extinta una mayor ventaja aerodinámica y puede permanecer mayor tiempo volando que las otras aves.

Tanto la carga alar y la relación de aspecto de $P$. chilensis, le ofrecen una mayor velocidad de planeo y un mayor tiempo de vuelo que las grandes aves marinas actuales, con lo cual podía cubrir mayores distancias que estas. Ante una eventual escases, esta ventaja le habría favorecido en la obtención del recurso alimenticio en zonas alejadas. La presencia de Pelagornithidae en extensas regiones marinas, se sostiene en que la especie encontrada en Atacama, Chile, también habría habitado las costas peruanas.

Estos comportamientos de vuelo descritos por Pennycuick (1982) están bien diferenciados por el tamaño de estas aves marinas; entonces, podemos hipotetizar que el ave fósil por el tamaño y morfología similar al albatros, 
tendría un tipo de vuelo semejante al de los albatros gigantes actuales. Asimismo, se puede hipotetizar que, considerando a Pelagornis un ave que principalmente planeaba y por su tamaño y forma del ala, las afirmaciones de Richardson (2011) para los albatros planeadores, podría aplicarse a $P$. chilensis, como la de usar la cizalladura del viento para la extracción de energía.

\section{CONCLUSIONES}

El estudio de las aves gigantes extintas ha revelado que poseían una gran adaptación y eficiencia de vuelo (Ksepka, 2014; Cannell, 2020; Chatterjee et al., 2007; Pennycuick, 1996). La importancia de las investigaciones trasciende el vuelo de estos grupos de aves y adquieren implicancias paleoclimáticas, de reconstrucción de las características físicas de la atmósfera del pasado (Cannel, 2020; Pennycuick, 1996). Este trabajo confirma ese conocimiento con la aerodinámica de vuelo de Pelagornis chilensis, no solo por la gran capacidad de planeo y la eficiente estrategia de vuelo aprovechando las térmicas ("soaring"), similar a los albatros actuales, como lo afirma Kpsepka (2014), sino por el desarrollo de altas velocidades correlacionado con una adecuada potencia para el vuelo, que va desde una velocidad mínima de vuelo de alrededor de $50 \mathrm{~km} / \mathrm{h}$ hasta una velocidad con el máximo rango de $85 \mathrm{~km} / \mathrm{h}$, pudiendo desarrollar velocidades de forrajeo asociadas a tormentas de más de $160 \mathrm{~km} / \mathrm{h}$. Estas velocidades superan en alrededor del $30 \%$ las velocidades del albatros de cabeza gris, Thalassarche chrysostoma. Además, la potencia para el avance (potencia parásita) del ave extinta se calcula en alrededor de 13 veces la misma potencia del mencionado albatros. P. chilensis también supera en velocidad al ave existente de mayor envergadura, el albatros Diomedea exulans, en alrededor del 17\%. Los valores de velocidad y razón de potencia parásita obtenidos van acorde con los altos valores de carga alar y relación de aspecto de $P$. chilensis hallados en este trabajo. Con esas velocidades, valor de carga alar y de relación de aspecto, el ave extinta podía volar a grandes distancias, mayores a las recorridas por las aves marinas actuales, ofreciéndole ventajas alimenticias cuando el recurso escaseaba en determinadas regiones.

Además, dada la morfología semejante y gran tamaño, como los albatros actuales, se hipotetiza que Pelagornis chilensis casi nunca batía sus alas, excepto en vientos muy leves, por lo que podía pasar todo el tiempo en vuelo planeado. Durante el vuelo sostenido sin batir las alas durante tanto tiempo, Pelagornis, al igual que los grandes albatros actuales, extraía energía del viento, probablemente usando cizalladuras (o cortes) del viento para ganar energía. También, el ave fósil habría volado contra el viento siguiendo un recorrido en zigzag $\mathrm{y}$, tal como los albatros y petreles más grandes, extrayendo energía de la gradiente vertical de la velocidad del viento, como en las subidas contra el viento.

\section{AGRADECIMIENTOS}

Agradezco a mi amigo Aldo Benites del Departamento de Paleontología de Vertebrados del Museo de Historia Natural de la UNMSM, por la traducción al inglés del resumen.

\section{REFERENCIAS}

Anderson, J. 2001. Fundamentals of Aerodynamics. 3ra Edición. McGrawHill

Averianov, A.O., Panteleyev, A.V., Potapova, O.R. \& Nessov, L.A. 1991. Bony-toothed birds (Aves: Pelecaniformes: Odontopterygia) of the late Paleocene and Eocene of the western margin of ancient Asia. Trudy Zoologicheskogo Instituta, Akademiya Nauk SSSR, 239, 3-12 [in Russian].

Bourdon, E. 2005. Osteological evidence for sister group relationship between pseudo-toothed birds (Aves: Odontopterygiformes) and waterfowls (Anseriformes). Naturwissenschaften, 92, 586-591; doi: 10.1007/s00114005-0047-0.

Brooke, M. de L. 2002. Seabird systematics and distribution: a review of current knowledge. In: Biology of Marine Birds (eds Schreiber, E.A. \& Burger, J.). CRC Press; doi: 10.1201/9781420036305.

Butler, P. 2016. The physiological basis of bird flight. Philosophical Transactions of the Royal Society B Biological Sciences, 371(1704):20150384; doi: 10.1098/ rstb.2015.0384.

Cannell, A. 2020. Too big to fly? An engineering evaluation of the fossil biology of the giant birds of the Miocene in relation to their flight limitations, constraining the minimum air pressure at about 1.3 bar. Animal Biology, Advance Articles, doi: 10.1163/15707563-bja10001.

Catry, P., Phillips, R. \& Croxall, J. 2004. Sustained fast travel by a gray-headed albatros (Thalassarche chrysostoma) riding an Antarctic storm. The Auk, 121, 1208-1213; doi: 10.1093/auk/121.4.1208.

Chatterjee, S., Templin, J. \& Campbell, E. 2007. The aerodynamics of Argentavis, the world's largest flying bird from the Miocene of Argentina. Proceedings of the National Academy of Sciences, 104, 12398-12403; doi: 10.1073/pnas.0702040104.

Chávez, M. \& Stucchi, M. 2002. El registro de Pelagornithidae del Pacífico sudeste. In: Actas del Primer Congreso Latinoamericano de Paleontología de Vertebrados, 26; Santiago. 
Chávez, M., Stucchi, M. \& Urbina, M. 2007. El registro de Pelagornithidae (Aves: Pelecaniformes) y la avifauna neógena del Pacífico sudeste. Bulletin de l'Institut Français d'Études Andines, 36, 175-197; doi: 10.4000/ bifea.3780.

Croxall, J.P., Silk, J.R.D., Phillips, R.A., Afanasyev, V. \& Briggs, D.R. 2005. Global circumnavigations: tracking year-round ranges of nonbreeding albatrosses. Science 307, 249-250; doi: 10.1126/science.1106042.

Feduccia, A. 1999. The Origin and Evolution of Birds. Yale University Press.

Fürbringer, M. 1888. Untersuchungen zur Morphologie und Systematik der Vögel, zugleich ein Beitrag zur Anatomie der Stützund Bewegungsorgane, 2 vols. van Holkema, Amsterdam.

González-Barba, G., Schwennicke, T., Goedert, J.L. \& Barnes, L.G. 2002. Earliest Pacific Basin record of the Pelagornithidae (Aves: Pelecaniformes). Journal of Vertebrate Paleontology, 22, 722-725; doi: 10.1671/0272-4634(2002)022[0722:EPBROT]2.0.CO;2.

Jou, D., Llebot, J. \& García, C. 2009. Física para Ciencias de la Vida. 2 da Ed. McGraw Hill.

Kardong, K. 1998. Vertebrates, Comparative Anatomy, Function, Evolution. 2da Ed. McGraw Hill.

Ksepka, D. 2014. Flight performance of the largest volant bird. Proceedings of the National Academy of Sciences, 111, 10624-10629; doi: 10.1073/pnas.1320297111.

Longrich, N. 2006. Structure and function of hindlimb feathers in Archaeopteryx lithographica. Paleobiology, 32, 417-431; doi: 10.1666/04014.1.

Marden, J.H. 1994. From damselflies to pterosaurs: how burst and sustainable flight performance scale with size. American Journal of Physiology, 266, R1077-R1084; doi: 10.1152/ajpregu.1994.266.4.R1077.

Marquardt, C., Blanco, N., Godoy, E., Lavenu, A., Ortlieb, L., Marchant, M. \& Guzmán, N. 2000. Estratigrafía del Cenozoico superior en el área de Caldera $\left(26^{\circ} 45^{\prime}-28^{\circ} \mathrm{S}\right)$, III Región de Atacama, Chile. IX Congreso Geológico Chileno V1, 504-508.

Matsuoka, H., Sakakura, F. \& Ohe, F. 1998. A Miocene pseudodontorn (Pelecaniformes: Pelagornithidae) from the Ichishi Group of Misato, Mie Prefecture, Central Japan. Paleontological Research, 2, 246-252.

Mayr, G. \& Rubilar-Rogers, D. 2010. Osteology of a new giant bony-toothed bird from the Miocene of Chile, with a revision of the taxonomy of Neogene Pelagornithidae. Journal of Vertebrate Paleontology, 30, 1313-1330; doi: 10.1080/02724634.2010.501465.

Mayr, G., Hazeboet, C., Dantas, P. \& Cachao, M. 2008. A sternum of a very large bony-toothed bird (Pelagornithidae) from the Miocene of Portugal. Journal of Vertebrate Paleontology, 28, 762-769; doi: 10.1671/0272-4634(2008)28[762:ASOAVL]2.0.CO;2.

McKee, J.W.A. 1985. A pseudodontorn (Pelecaniformes: Pelagornithidae) from the Middle Pliocene of Hawera, Taranaki, New Zealand. New Zealand Journal of Zoology, 12, 181-184; doi: 10.1080/03014223.1985.10428278.
Meseguer, J. \& Sanz-Andrés, A. 2007. Aerodinámica del Vuelo: Aves y Aeronaves. Aena.

Muizon, C. de \& Devries, T. 1985. Geology and paleontology of late Cenozoic marine deposits in Sacaco area (Peru). Geologische Rundschau, 74, 547-563; doi: 10.1007/ BF01821211.

Norberg, U.M. 2002. Structure, form, and function of flight in engineering and the living world. Journal of Morphology, 252, 52-81; doi: 10.1002/jmor.10013.

Olson, S.L. 1985. The fossil record of birds. In: Avian Biology, Volume 8 (eds Farner, D.S., King, J.R. \& Parkes, K.C.). Academic Press, New York. 79-238.

Olson, S.L. 1999. Early Eocene birds from Eastern North America: A faunule from the Nanjemoy Formation of Virginia. In: Early Eocene Vertebrates and Plants from the Fisher/Sullivan Site (Nanjemoy Formation) Stafford County, Virginia (eds.Weems, R.E. \& Grimsley, G.J.). Virginia Division of Mineral Resources Publication, $152,123-132$

Olson, S.L. \& Rasmussen, P.C. 2001. Miocene and Pliocene birds from the Lee Creek Mine, North Carolina. Smithsonian Contributions to Paleobiology, 90, 233-365; doi: 10.5479/SI.00810266.90.1.

Pennycuick, C.J. 1975. Mechanics of Flight. In: Avian Biology, Volume 5 (eds Farner, D.S. \& King, J.R.). New York, Academic Press. 1-75.

Pennycuick, C.J. 1982. The flight of petrels and albatrosses (Procellariiformes), observed in South Georgia and its vicinity. Philosophical Transactions of the Royal Society of London, Series B, 300, 75-106; doi: 10.1098/ rstb.1982.0158.

Pennycuick, C.J. 1986. Mechanical constraints on the evolution of flight. Biodiversity Heritage Library, Memoirs of the California Academy of Sciences, 7, 8-10; (1986-1988): https://www.biodiversitylibrary.org/ item/53408.

Pennycuick, C.J. 1987. Flight of Seabirds. In: Seabirds: Feeding Ecology and Role in Marine Ecosystems (ed. Croxall, J.P.). Cambridge University Press. 43-62.

Pennycuick, C.J. 1996. Stress and strain in the flight muscles as constraints on the evolution of flying animals. Journal of Biomechanics, 29, 577-581; doi: 10.1016/00219290(95)00137-9.

Pennycuick, C.J. 2008. Modelling the Flying Bird. Theoretical Ecology Series, Elsevier.

Pizzey, G. \& Knight, F. 1999. The Graham Pizzey and Frank Knight Field Guide to the Birds of Australia. Angus and Robertson. Australia.

Rayner, J.M. 1988. Form and function in avian flight. Current Ornithology, 5, 1-66.

Richardson, P. 2011. How do albatrosses fly around the world without flapping their wings? Progress in Oceanography, 88, 46-58; doi: 10.1016/j.pocean.2010.08.001.

Rincón, A.D. \& Stucchi, M. 2003. Primer registro de la familia Pelagornithidae (Aves: Pelecaniformes) para Venezuela. Boletín de la Sociedad Venezolana de Espeleología, 37, 27-30. 
Rincón, A.D. \& Stucchi, M. 2005. Primer registro de Pelagornithidae del Mioceno de Venezuela. Revista Venezolana de Espeleología, 37, 27-30.

Rojo, M. 1985. Un aporte al conocimiento del terciario marino: Formación Bahía Inglesa. Actas IV Congreso Geológico Chileno, 1-514.

Safina, C. 2002. Eye of the Albatross: Visions of Hope and Survival. Henry Holt \& Company, New York.

Safina, C. 2007. On the wings of the albatross. National Geographic, 212, 96-113.

Schulenberg, T., Stotz, D., Lane, D., O’Neill, J. \& Parker, T. 2010. Birds of Peru. Princeton Field Guides.

Serrano, F.J. \& Chiappe, L.M. 2017. Aerodynamic modelling of a Cretaceous bird reveals thermal soaring capabilities during early avian evolution. Journal of the Royal Society Interface, 14, 20170182; doi: 10.1098/rsif.2017.0182.

Serrano, F.J., Palmqvist, P., Chiappe, L.M. \& Sanz, J.L. 2017. Inferring flight parameters of Mesozoic avians through multivariate analyses of forelimb elements in their living relatives. Paleobiology, 43, 144-169; doi: 10.1017/pab.2016.35.

Serrano, F.J., Chiappe, L.M., Palmqvist, P., Figueirido, B., Marugan-Lobon, J. \& Sanz, J.L. 2018. Flight reconstruction of two European Enantiornithines (aves, pygostylia) and the achievement of bounding flight in early Cretaceous birds. Palaeontology, 61, 359-368, doi; 10.1111/pala.12351.

Tennekes, H. 1996. The Simple Science of Flight: from Insects to Jumbo Jets. MIT Press, Cambridge.

Tonni, E.P. \& Tambussi, C.P. 1985. Nuevos restos de Odontopterygia (Aves: Pelecaniformes) del Terciario temprano de Antártida. Ameghiniana, 21, 121-124.

Videler, J. 2005. Avian Flight. Oxford Ornithology Series, Oxford University Press.

Villar, R., López, C. \& Cussó, F. 2013. Fundamentos Físicos de los Procesos Biológicos. Volumen 2. Editorial Club Universitario.

Viscor, G. \& Fuster, J.F. 1987. Relationships between morphological parameters in birds with different flying habits. Comparative Biochemistry and Physiology A, 87, 231-249; doi:10.1016/0300-9629(87)90118-6.

Vogel, S. 1996. Life in Moving Fluids: the Physical Biology of Flow. 2nd Edition. Princeton, Princeton University Press.

Walsh, S.A. \& Hume, J.P. 2001. A new Neogene marine avian assemblage from north-central Chile. Journal of Vertebrate Paleontology, 21, 484-491; doi: 10.1671/0272-4634(2001)021[0484:ANNMAA]2.0.CO;2. 


\section{APÉNDICE}

\section{Desarrollo Matemático}

Se toman los siguientes datos:

\section{Pelagornis chilensis:}

Envergadura B $=520 \mathrm{~cm}(5,2 \mathrm{~m})$ (Mayr et al., 2010) Peso: 16 - 29 kg (Mayr et al., 2010). Media: $22,5 \mathrm{~kg}$ Velocidad: ? (denotaremos la velocidad del Pelagornis como $v_{p}$ )

Área del ala de Pelagornis, $S_{p}: 1,33 \mathrm{~m}^{2}$ (método del papel milimetrado translúcido o cuadrícula transparente)

\section{Thalassarche chrysostoma:}

Se toman los valores de Pennycuick (1982):

$\mathrm{B}=2,18 \mathrm{~m}$

Masa $=3,79 \mathrm{~kg}$

$S_{a}=0,352 \mathrm{~m}^{2}$

$V_{m p}=10,9 \mathrm{~m} / \mathrm{s}(39,2 \mathrm{~km} / \mathrm{h})$ (velocidad de mínima potencia)

$V_{m r}=17,9 \mathrm{~m} / \mathrm{s}(64,4 \mathrm{~km} / \mathrm{h})$ (velocidad con el máximo rango)

$V_{b g}=14 \mathrm{~m} / \mathrm{s}(50,4 \mathrm{~km} / \mathrm{h})$ (velocidad con la tasa máxima de planeo, 22)

De Catry et al. (2004) se utiliza:

Velocidad $\geq 127 \mathrm{~km} / \mathrm{h}$ o $35,3 \mathrm{~m} / \mathrm{s}$ (velocidad media real de forrajeo asociada a una tormenta en la Antártida). En general, denotaremos la velocidad del albatros de cabeza gris como $v_{a}$

\section{Diomedea exulans:}

Se toman los valores de Pennycuick (1982):

$\mathrm{B}=3,03 \mathrm{~m}$

Masa $=8,73 \mathrm{~kg}$

$S_{a}=0,611 \mathrm{~m}^{2}$

$V_{m p}=12,2 \mathrm{~m} / \mathrm{s}(43,9 \mathrm{~km} / \mathrm{h})($ velocidad de mínima potencia)

$V_{m r}=20,0 \mathrm{~m} / \mathrm{s}(72 \mathrm{~km} / \mathrm{h})$ (velocidad con el máximo rango)

$V_{b g}=15,7 \mathrm{~m} / \mathrm{s}(56,5 \mathrm{~km} / \mathrm{h})($ velocidad con la tasa máxima de planeo, 23,2)

En general, denotaremos la velocidad de este albatros como $v_{D}$
Densidad del aire:

$\rho_{\text {aire }}=1,2254 \mathrm{~kg} / \mathrm{m}^{3}$ (promedio) a $0 \mathrm{~m}$ de altitud $\rho_{\text {aire }}=1,1886 \mathrm{~kg} / \mathrm{m}^{3}$ (promedio) a $305 \mathrm{~m}$ de altitud

Se toma la densidad del aire a $0 \mathrm{~m}$ de altitud, considerando que los vuelos de los albatros y petreles de gran tamaño estudiados por Pennycuick (1982) van desde los 3,2 a 7,7 $\mathrm{m}$ sobre el nivel del mar.

\section{Por la fórmula de sustentación (2): velocidad mínima del pelagornítido}

$$
\begin{gathered}
16 \times 10=1,33 \times 0,75 \times 1,2254 v_{p}^{2} \\
29 \times 10=1,33 \times 0,75 \times 1,2254 v_{p}^{2} \\
11,4 \frac{\mathrm{m}}{\mathrm{s}}<v_{p}<15,4 \frac{\mathrm{m}}{\mathrm{s}} \\
41 \frac{\mathrm{km}}{\mathrm{h}}<v_{p}<55,4 \frac{\mathrm{km}}{\mathrm{h}}
\end{gathered}
$$

Con masa promedio:

$$
\begin{gathered}
22,5 \times 10=1,33 \times 0,75 \times 1,2254 v_{p}{ }^{2} \\
v_{p}=13,6 \frac{\mathrm{m}}{\mathrm{s}} \quad\left(v_{p}=48,9 \frac{\mathrm{km}}{\mathrm{h}}\right)
\end{gathered}
$$

2. Por la fórmula de sustentación (2): velocidad mínima del albatros

$$
\begin{aligned}
3,79 \times 10 & =0,352 \times 0,75 \times 1,2254 v_{a}{ }^{2} \\
v_{a} & =10,8 \frac{\mathrm{m}}{\mathrm{s}}\left(38,9 \frac{\mathrm{km}}{\mathrm{h}}\right)
\end{aligned}
$$

3. Velocidad de $\boldsymbol{P}$. chilensis por alometría con respecto a $T$. chrysostoma en función de la longitud $L$ del ave

\subsection{Velocidad media real de forrajeo asociada a una tormenta en la Antártida}

\footnotetext{
Albatros:

$v_{a} \geq 127 \mathrm{~km} / \mathrm{h}$ 1982)

\section{Pelagornítido:}

$v_{p}=? \mathrm{~km} / \mathrm{h}$

Envergadura $\left(B=L_{p}\right): 520 \mathrm{~cm}$

De $v_{p} \alpha \sqrt{L}$
}

Vamos a suponer que la envergadura $\left(B=L_{a}\right)$ del albatros de Catry et al. (2004) es: $218 \mathrm{~cm}$ (Pennycuick, 


$$
\begin{gathered}
v_{p} \geq\left(\frac{L_{p}}{L_{a}}\right)^{\frac{1}{2}} v_{a} \geq\left(\frac{520}{218}\right)^{\frac{1}{2}} v_{a} \geq \sqrt{2,4} \times 127 \\
\geq 196,7 \frac{\mathrm{km}}{\mathrm{h}}\left(54,6 \frac{\mathrm{m}}{\mathrm{s}}\right)
\end{gathered}
$$

\subsection{Velocidad de mínima potencia}

\section{Albatros:}

$V_{m p}=10,9 \mathrm{~m} / \mathrm{s}(39,2 \mathrm{~km} / \mathrm{h})$

Envergadura $\left(B=L_{a}\right): 218 \mathrm{~cm}$ (Pennycuick, 1982)

\section{Pelagornítido:}

$v_{p}=? \mathrm{~km} / \mathrm{h}$

Envergadura $\left(B=L_{p}\right): 520 \mathrm{~cm}$

De $v_{p} \alpha \sqrt{L}$

$$
\begin{gathered}
v_{p}=\left(\frac{L_{p}}{L_{a}}\right)^{\frac{1}{2}} v_{a}=\left(\frac{520}{218}\right)^{\frac{1}{2}} v_{a}=\sqrt{2,4} \times 39,2 \\
=60,7 \frac{\mathrm{km}}{\mathrm{h}}\left(16,9 \frac{\mathrm{m}}{\mathrm{s}}\right)
\end{gathered}
$$

\subsection{Velocidad con el máximo rango}

\section{Albatros:}

$V_{m r}=17,9 \mathrm{~m} / \mathrm{s}(64,4 \mathrm{~km} / \mathrm{h})$

Envergadura $\left(B=L_{a}\right): 218 \mathrm{~cm}$ (Pennycuick, 1982)

\section{Pelagornítido:}

$v_{p}=? \mathrm{~km} / \mathrm{h}$

Envergadura $\left(B=L_{p}\right): 520 \mathrm{~cm}$

De $v_{p} \alpha \sqrt{L}$

$$
\begin{gathered}
v_{p}=\left(\frac{L_{p}}{L_{a}}\right)^{\frac{1}{2}} v_{a}=\left(\frac{520}{218}\right)^{\frac{1}{2}} v_{a}=\sqrt{2,4} \times 64,4 \\
=99,8 \frac{\mathrm{km}}{\mathrm{h}}\left(27,7 \frac{\mathrm{m}}{\mathrm{s}}\right)
\end{gathered}
$$

\subsection{Velocidad con la tasa máxima de planeo}

Albatros:

$V_{b g}=14 \mathrm{~m} / \mathrm{s}(50,4 \mathrm{~km} / \mathrm{h})$

Envergadura $\left(B=L_{a}\right): 218 \mathrm{~cm}$ (Pennycuick, 1982)

\section{Pelagornítido:}

$v_{p}=? \mathrm{~km} / \mathrm{h}$

Envergadura $\left(B=L_{p}\right): 520 \mathrm{~cm}$

$$
\begin{gathered}
v_{p}=\left(\frac{L_{p}}{L_{a}}\right)^{\frac{1}{2}} v_{a}=\left(\frac{520}{218}\right)^{\frac{1}{2}} v_{a}=\sqrt{2,4} \times 50,4 \\
=78,1 \frac{\mathrm{km}}{\mathrm{h}}\left(21,7 \frac{\mathrm{m}}{\mathrm{s}}\right)
\end{gathered}
$$

\section{Por alometría: velocidad en función de la masa, utilizando formula (6)}

Masas de P. chilensis: $m_{p}=16 \mathrm{~kg}$ y $29 \mathrm{~kg}$, y $22,5 \mathrm{~kg}$ (promedio); masa del albatros $m_{a}=3,79 \mathrm{~kg}$

$$
\begin{aligned}
v_{p} \alpha m_{p}^{\frac{1}{6}} & =>v_{p}=\left(\frac{16}{3,79} m_{a}\right)^{\frac{1}{6}}=\left(4,22 m_{a}\right)^{\frac{1}{6}} \\
& =(4,22)^{\frac{1}{6}} m_{a^{\frac{1}{6}}}=1,27 m_{a^{\frac{1}{6}}}
\end{aligned}
$$

entonces $v_{p}=1,27 m_{a}{ }^{\frac{1}{6}}$ pero $v_{a} \alpha m_{a^{\frac{1}{6}}}=>\boldsymbol{v}_{\boldsymbol{p}}$ $=1,27 v_{a}$

$$
\begin{aligned}
v_{p} \alpha m_{p^{\frac{1}{6}}} & =>v_{p}=\left(\frac{29}{3,79} m_{a}\right)^{\frac{1}{6}}=\left(7,65 m_{a}\right)^{\frac{1}{6}} \\
& =(7,65)^{\frac{1}{6}} m_{a}^{\frac{1}{6}}=1,4 m_{a}^{\frac{1}{6}}
\end{aligned}
$$

entonces $v_{p}=1,4 m_{a}^{\frac{1}{6}}$ pero $v_{a} \alpha m_{a^{\frac{1}{6}}}=>v_{p}$

$$
=1,4 v_{a}
$$

Con masa promedio:

$$
\begin{aligned}
& v_{p} \alpha m_{p}^{\frac{1}{6}}=>v_{p}=\left(\frac{22,5}{3,79} m_{a}\right)^{\frac{1}{6}}=\left(5,94 m_{a}\right)^{\frac{1}{6}} \\
& =(5,94)^{\frac{1}{6}} m_{a^{\frac{1}{6}}}=1,35 m_{a}^{\frac{1}{6}} \\
& =1,35 v_{a}
\end{aligned}
$$

(La velocidad de $P$. chilensis es 1,35 veces la velocidad del albatros T. Chrysostoma)

\section{Por alometría: potencia parásita (10) e inducida (11) y fuerza muscular (12)}

Usamos: $P_{\text {par }} \alpha L^{\frac{7}{2}}$ y $P_{\text {ind }} \alpha L^{\frac{7}{2}}$

El Pelagornítido necesita una potencia: $P_{\text {par }} \alpha(2,39)^{\frac{7}{2}}=$ 21,1 veces la del ave actual, igual valor para $P_{\text {ind }} ; L=$ $\frac{520 \mathrm{~cm}}{218 \mathrm{~cm}}=2,39$

La fuerza muscular es proporcional a $L^{2}\left(F \alpha L^{2}\right)$, entonces: $F=(2,39)^{2}=5,7$ veces la del albatros. 


\section{Modelo de flujo para el ala}

Usamos: $R_{\text {ewing }}=\frac{v_{t} C_{m}}{v}$, con $v=\frac{\mu}{\rho}$ y $C_{m}=\frac{S}{B}$ (cuerda del ala)

\section{Pelagornítido:}

Con velocidad media de forrajeo de albatros asociada a tormenta: $v=127 \frac{\mathrm{km}}{\mathrm{h}}$

$$
\operatorname{Re}=\frac{\left(35,3 \frac{\mathrm{m}}{\mathrm{s}}\right)\left(\frac{1,33 \mathrm{~m}^{2}}{5,2 \mathrm{~m}}\right)\left(1,2254 \frac{\mathrm{kg}}{\mathrm{m}^{3}}\right)}{18.08 \times 10^{-6} \mathrm{~kg} / \mathrm{ms}}=611931
$$

Con velocidad calculada asociada a tormenta: $v=$ $167 \frac{\mathrm{km}}{\mathrm{h}}$

$$
\operatorname{Re}=\frac{\left(46,4 \frac{\mathrm{m}}{\mathrm{s}}\right)\left(\frac{1,33 \mathrm{~m}^{2}}{5,2 \mathrm{~m}}\right)\left(1,2254 \frac{\mathrm{kg}}{\mathrm{m}^{3}}\right)}{18.08 \times 10^{-6} \mathrm{~kg} / \mathrm{ms}}=804351
$$

Con velocidad de albatros con la mínima potencia: $v=$ $39,2 \frac{\mathrm{km}}{\mathrm{h}}$

$$
\operatorname{Re}=\frac{\left(10,9 \frac{\mathrm{m}}{\mathrm{s}}\right)\left(\frac{1,33 \mathrm{~m}^{2}}{5,2 \mathrm{~m}}\right)\left(1,2254 \frac{\mathrm{kg}}{\mathrm{m}^{3}}\right)}{18.08 \times 10^{-6} \mathrm{~kg} / \mathrm{ms}}=188953
$$

Con velocidad calculada con la mínima potencia: $v=$ $50,5 \frac{\mathrm{km}}{\mathrm{h}}$

$$
\operatorname{Re}=\frac{\left(14 \frac{\mathrm{m}}{\mathrm{s}}\right)\left(\frac{1,33 \mathrm{~m}^{2}}{5,2 \mathrm{~m}}\right)\left(1,2254 \frac{\mathrm{kg}}{\mathrm{m}^{3}}\right)}{18.08 \times 10^{-6} \mathrm{~kg} / \mathrm{ms}}=242692
$$

\section{Albatros:}

Con velocidad media de forrajeo asociada a tormenta: $v=127 \frac{\mathrm{km}}{\mathrm{h}}$

$$
\operatorname{Re}=\frac{\left(35,3 \frac{\mathrm{m}}{\mathrm{s}}\right)\left(\frac{0,352 \mathrm{~m}^{2}}{2,18 \mathrm{~m}}\right)\left(1,2254 \frac{\mathrm{kg}}{\mathrm{m}^{3}}\right)}{\frac{18.08 \times 10^{-6} \mathrm{~kg}}{\mathrm{~ms}}}=386314
$$

Con velocidad de mínima potencia: $v=39,2 \frac{\mathrm{km}}{\mathrm{h}}$

$$
\operatorname{Re}=\frac{\left(10,9 \frac{\mathrm{m}}{\mathrm{s}}\right)\left(\frac{0,352 \mathrm{~m}^{2}}{2,18 \mathrm{~m}}\right)\left(1,2254 \frac{\mathrm{kg}}{\mathrm{m}^{3}}\right)}{18.08 \times 10^{-6} \mathrm{~kg} / \mathrm{ms}}=119287
$$

\section{Velocidades de Diomedea exulans por alometría con respecto a $T$. chrysostoma}

\subsection{En función de la longitud, utilizando fórmula (5)}

\subsubsection{Velocidad de mínima potencia}

\section{T. chrysostoma:}

$V_{m p}=10,9 \mathrm{~m} / \mathrm{s}(39,2 \mathrm{~km} / \mathrm{h})$

Envergadura $\left(B=L_{a}\right): 218 \mathrm{~cm}$ (Pennycuick, 1982)

\section{Diomedea exulans:}

$v_{D}=? \mathrm{~km} / \mathrm{h}$

Envergadura $\left(B=L_{D}\right): 303 \mathrm{~cm}$

De $v_{D} \alpha \sqrt{L}$

$$
\begin{gathered}
v_{D}=\left(\frac{L_{D}}{L_{a}}\right)^{\frac{1}{2}} v_{a}=\left(\frac{303}{218}\right)^{\frac{1}{2}} v_{a}=\sqrt{1,39} \times 39,2 \\
=46,2 \frac{\mathrm{km}}{\mathrm{h}}\left(12,8 \frac{\mathrm{m}}{\mathrm{s}}\right)
\end{gathered}
$$

\subsubsection{Velocidad con el máximo rango}

\section{T. chrysostoma:}

$V_{m r}=17,9 \mathrm{~m} / \mathrm{s}(64,4 \mathrm{~km} / \mathrm{h})$

Envergadura $\left(B=L_{a}\right): 218 \mathrm{~cm}$ (Pennycuick, 1982)

\section{Diomedea exulans:}

$v_{D}=? \mathrm{~km} / \mathrm{h}$

Envergadura $\left(B=L_{D}\right): 303 \mathrm{~cm}$

De $v_{D} \alpha \sqrt{L}$

$$
\begin{gathered}
v_{D}=\left(\frac{L_{D}}{L_{a}}\right)^{\frac{1}{2}} v_{a}=\left(\frac{303}{218}\right)^{\frac{1}{2}} v_{a}=\sqrt{1,39} \times 64,4 \\
=75,9 \frac{\mathrm{km}}{\mathrm{h}}\left(21,1 \frac{\mathrm{m}}{\mathrm{s}}\right)
\end{gathered}
$$

\subsubsection{Velocidad con la tasa máxima de planeo}

T. chrysostoma:

$V_{m r}=14 \mathrm{~m} / \mathrm{s}(50,4 \mathrm{~km} / \mathrm{h})$

Envergadura $\left(B=L_{a}\right): 218 \mathrm{~cm}$ (Pennycuick, 1982)

\section{Diomedea exulans:}

$v_{D}=? \mathrm{~km} / \mathrm{h}$

Envergadura $\left(B=L_{D}\right): 303 \mathrm{~cm}$

De $v_{D} \alpha \sqrt{L}$

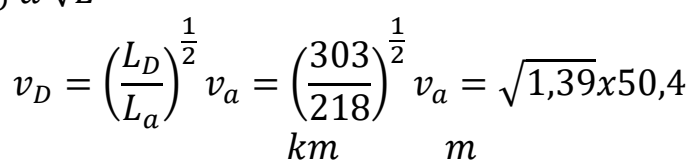




\subsection{En función de la masa, utilizando la fórmula (6)}

Masas: $m_{D}=8,73 \mathrm{~kg} ; m_{a}=3,79 \mathrm{~kg}$

$$
\begin{aligned}
v_{D} \alpha m_{D}^{\frac{1}{6}} & =>v_{D}=\left(\frac{8,73}{3,79} m_{a}\right)^{\frac{1}{6}}=\left(2,3 m_{a}\right)^{\frac{1}{6}} \\
& =(2,3)^{\frac{1}{6}} m_{a^{\frac{1}{6}}}=1,15 m_{a^{\frac{1}{6}}} \\
\text { entonces } v_{D}= & 1,15 m_{a}^{\frac{1}{6}} \text { pero } v_{a} \alpha m_{a^{\frac{1}{6}}}=>\boldsymbol{v}_{\boldsymbol{D}} \\
& =\mathbf{1}, \mathbf{1 5} \boldsymbol{v}_{\boldsymbol{a}}
\end{aligned}
$$

Para: $v_{a}=39,2 \frac{\mathrm{km}}{\mathrm{h}}$ obtenemos $v_{D}=45,1 \frac{\mathrm{km}}{\mathrm{h}}$

$$
\begin{aligned}
& v_{a}=50,4 \frac{\mathrm{km}}{\mathrm{h}} \text { obtenemos } v_{D}=57,9 \frac{\mathrm{km}}{\mathrm{h}} \\
& v_{a}=64,4 \frac{\mathrm{km}}{\mathrm{h}} \text { obtenemos } v_{D}=74,1 \frac{\mathrm{km}}{\mathrm{h}}
\end{aligned}
$$

\section{Por fórmula de sustentación (2): velocidad mínima de Diomedea exulans}

$$
\begin{aligned}
8,73 \times 10 & =0,611 \times 0,75 \times 1,2254 v_{D}{ }^{2} \\
v_{D} & =12,5 \frac{\mathrm{m}}{\mathrm{s}}\left(45 \frac{\mathrm{km}}{\mathrm{h}}\right)
\end{aligned}
$$

\section{Razón potencia parásita $P$. chilensis/potencia parásita $T$. chrysostoma con la fórmula (7)}

Dada que la fórmula (7) es más manejable, solo utilizaremos la potencia parásita. De:

$$
P_{\text {par }}=\frac{1}{2} v^{3} \rho C_{a} S_{t}
$$

observamos que no tenemos el valor de $S_{t}$ para ninguna de las dos aves, que es la sección transversal del cuerpo en la dirección del movimiento. Entonces se tomará la única medida de que disponemos, la envergadura, que la llamaremos L para el pelagornítido y L' para el albatros. Luego, consideraremos para la sección transversal un círculo de radio $r$ para el pelagornítido y $\mathrm{r}^{\prime}$ para el albatros. Se hallará la razón de la dimensión lineal entre $\mathrm{L}_{\text {y L'}}$, que estará dada por:

$$
\frac{L}{L^{\prime}}=K
$$

Asumiremos que $\mathrm{K}$ también es la razón entre $\mathrm{r}$ y $\mathrm{r}^{\prime}$, por ser ambas, L y r, lineales, es decir, en la misma proporción que aumenta la envergadura aumenta el radio de la sección transversal del cuerpo. Entonces, esta relación la denotamos de la siguiente manera:

$$
\frac{r}{r^{\prime}}=K
$$

A continuación veremos la razón $\frac{P_{p a r}}{P^{\prime} \text { par }}$, es decir, la potencia parásita entre el pelagornítido y el albatros, con la siguiente expresión:

$$
\frac{P_{\text {par }}}{P_{\text {par }}^{\prime}}=\frac{1 / 2}{1 / 2} \frac{v^{3} \rho C_{a} S_{t}}{v^{\prime 3} \rho C^{\prime}{ }_{a} S_{t}^{\prime}}
$$

Asumiremos que la diferencia de los valores de los coeficientes de arrastre para ambas aves son despreciables, entonces la expresión se reduce a:

$$
\frac{P_{\text {par }}}{P_{\text {par }}^{\prime}}=\frac{v^{3} S_{t}}{v^{\prime} S_{t}^{\prime}}
$$

Siendo $S_{t}$ un círculo, la relación queda de la siguiente manera:

$$
\frac{P_{p a r}}{P_{p a r}^{\prime}}=\frac{v^{3} \pi r^{2}}{v^{\prime 3} \pi r^{\prime 2}}
$$

Cancelando $\pi$ y haciendo un arreglo por ley de exponentes, tenemos:

$$
\frac{P_{\text {par }}}{P_{\text {par }}^{\prime}}=\frac{v^{3}}{v^{\prime 3}}\left(\frac{r}{r^{\prime}}\right)^{2}
$$

Lo que es lo mismo que:

$$
\frac{P_{\text {par }}}{P_{\text {par }}^{\prime}}=\frac{v^{3}}{v^{\prime 3}}(K)^{2}
$$

Para $v$ se utilizará primero la velocidad de mínima potencia calculada por alometría en función de la masa del pelagornítido con la media del peso $(22,5 \mathrm{~kg})$, es decir, 52,9 $\mathrm{km} / \mathrm{h}$ (Tabla 4); y para el albatros la velocidad de mínima potencia de $39,2 \mathrm{~km} / \mathrm{h}$. Para $K$ se tomará la razón entre $5,2 \mathrm{~m}$ y $2,18 \mathrm{~m}(5,2 / 2,18)$, que es la envergadura del pelagornítido (Mayr et al., 2010) y la envergadura del albatros registrada por Pennycuick (1982). Entonces, obtenemos el siguiente valor:

$$
\frac{P_{\text {par }}}{P_{\text {par }}^{\prime}}=\frac{52,9^{3}}{39,2^{3}}(2,39)^{2}=14
$$


Lo que significa que la potencia parásita del pelagornítido es 14 veces la potencia parásita del albatros con las velocidades de mínima potencia.

\section{Carga alar y relación de aspecto}

Usamos:

Carga alar: $Q=\frac{m}{s}$

Relación de aspecto: $R=\frac{B^{2}}{S}$
10.2. Thalassarche chrysostoma

$\mathrm{Q}=3,79 \mathrm{~kg} / 0,352 \mathrm{~m}^{2}=10,77 \mathrm{~kg} / \mathrm{m}^{2}$

$\mathrm{R}=(2,18 \mathrm{~m})^{2} / 0,352 \mathrm{~m}^{2}=13,5$

\subsection{Diomedea exulans}

$\mathrm{Q}=8,73 \mathrm{~kg} / 0,611 \mathrm{~m}^{2}=14,29 \mathrm{~kg} / \mathrm{m}^{2}$

$\mathrm{R}=(3,03 \mathrm{~m})^{2} / 0,611 \mathrm{~m}^{2}=15,03$

\subsection{Pelagornis chilensis}

Con la media de la masa:

$\mathrm{Q}=22,5 \mathrm{~kg} / 1,33 \mathrm{~m}^{2}=16,9 \mathrm{~kg} / \mathrm{m}^{2}$

$\mathrm{R}=(5,2 \mathrm{~m})^{2} / 1,33 \mathrm{~m}^{2}=20,33$ 